\title{
NLL soft and Coulomb resummation for squark and gluino production at the LHC
}

\author{
P. Falgari, ${ }^{a}$ C. Schwinn ${ }^{b}$ and C. Wever ${ }^{a}$ \\ ${ }^{a}$ Institute for Theoretical Physics and Spinoza Institute, \\ Utrecht University, 3508 TD Utrecht, The Netherlands \\ ${ }^{b}$ Albert-Ludwigs Universität Freiburg, Physikalisches Institut, \\ D-79104 Freiburg, Germany \\ E-mail: P.Falgari@uu.nl, christian.schwinn@physik.uni-freiburg.de, \\ C.S.P.Wever@uu.nl
}

ABSTRACT: We present predictions of the total cross sections for pair production of squarks and gluinos at the LHC, including the stop-antistop production process. Our calculation supplements full fixed-order NLO predictions with resummation of threshold logarithms and Coulomb singularities at next-to-leading logarithmic (NLL) accuracy, including boundstate effects. The numerical effect of higher-order Coulomb terms can be as big or larger than that of soft-gluon corrections. For a selection of benchmark points accessible with data from the 2010-2012 LHC runs, resummation leads to an enhancement of the total inclusive squark and gluino production cross section in the 15-30\% range. For individual production processes of gluinos, the corrections can be much larger. The theoretical uncertainty in the prediction of the hard-scattering cross sections is typically reduced to the $\pm 10 \%$ level.

KEYwords: Supersymmetry Phenomenology, NLO Computations

ARXiv EPRINT: 1202.2260 


\section{Contents}

1 Introduction $\quad 1$

2 NLL resummation for squark and gluino production 3

2.1 LO and NLO results 4

2.2 Soft-gluon and Coulomb resummation 8

$\begin{array}{lll}2.3 & \text { Stop-antistop production } & 11\end{array}$

$\begin{array}{ll}2.4 \text { Scale choices } & 14\end{array}$

3 Numerical results $\quad 16$

$\begin{array}{lll}3.1 & \text { Squark and gluino production at NLL } & 17\end{array}$

$\begin{array}{ll}3.2 & \text { Benchmark points for SUSY searches at LHC } \\ 3.3 & 22\end{array}$

$\begin{array}{lll}3.3 & \text { Results for stop-antistop production } & 27\end{array}$

3.4 Comparison with Mellin-space results 30

4 Conclusions and outlook $\quad 32$

A NLL resummation for stop-antistop production 33

$\begin{array}{ll}\text { B Resummation functions } & 36\end{array}$

$\begin{array}{ll}\text { C Determination of } \mu_{s} \text { and } \boldsymbol{\beta}_{\text {cut }} & 37\end{array}$

\section{Introduction}

Despite the good agreement of the Standard Model (SM) with a wealth of experimental data, both empirical reasons (e.g. the observation of dark matter) and theoretical arguments (such as the naturalness problem and the desire for gauge coupling unification) point to physics beyond the SM. One of the most thoroughly studied extensions of the SM is Supersymmetry (SUSY). In particular the Minimal Supersymmetric Standard Model (MSSM) with $R$-parity conservation and superpartner masses at the $\mathrm{TeV}$ scale could provide a solution to the above issues. The search for SUSY at the TeV scale is therefore a central part of the physics program of the Large Hadron Collider (LHC) at CERN. In the context of the MSSM, and of any other $R$-parity conserving model, the supersymmetric partners of the SM particles are produced in pairs, and squarks and gluinos, coupling strongly to quarks and gluons, have typically the highest production rates. Experimental searches for SUSY have been performed at LEP, the Tevatron and the LHC in various final-state signatures, see [1] for a recent review. For squark- and gluino-pair production the tightest bounds generically arise from jets+missing energy signatures, where the two LHC experiments have set lower bounds on the mass of squarks and gluinos of about $800 \mathrm{GeV}-1 \mathrm{TeV}[2,3]$, 
depending on the precise underlying theoretical model assumed. Once upgraded to its nominal energy of $14 \mathrm{TeV}$ the LHC should be sensitive to squark and gluino masses of up to $3 \mathrm{TeV}[4]$.

SUSY searches and, if squarks and gluinos are discovered, the measurement of their properties rely on a precise theoretical understanding of the production mechanism and on accurate predictions of the observables used in the analysis. From the theoretical point of view, the simplest of such observables is the total production cross section, on which we will focus in this work. For QCD mediated processes, such as squark- and gluino-pair production, the Born cross section is notoriously affected by large theoretical uncertainties, such that the inclusion of at least the next term in the expansion in the strong coupling constant $\alpha_{s}$ is mandatory for a reliable prediction. Next-to-leading order (NLO) SUSY QCD corrections for production of squarks and gluinos were computed in [5] and implemented in the program PROSPINO [6, 7]. The corrections are large, up to $100 \%$ of the tree-level result, and lead to a significant reduction of the scale dependence of the cross section. Electroweak contributions were also investigated [8-13], but found to be much smaller than the QCD contributions, less than $5-10 \%$ of the Born result.

The size of the $\mathcal{O}\left(\alpha_{s}\right)$ corrections raises the question of the magnitude of unknown higher-order QCD corrections, and makes it desirable to include at least the dominant contributions beyond NLO. It is known that a non-negligible part of the full NLO corrections arises from the partonic threshold region, defined by the limit $\beta \equiv \sqrt{1-4 M^{2} / \hat{s}} \rightarrow 0$, with $M$ the average mass of the particles produced and $\hat{s}$ the partonic centre-of-mass energy. In the threshold region the partonic cross section is dominated by soft-gluon emission off the initial- and final-state coloured particles and by Coulomb interactions of the two non-relativistic heavy particles, which give rise to singular terms of the form $\alpha_{s} \ln ^{2,1} \beta$ and $\alpha_{s} / \beta$, respectively. These corrections can be resummed to all orders in $\alpha_{s}$, thus leading to improved predictions of the cross section and smaller theoretical uncertainties. Note that to obtain the total hadronic cross section, the partonic cross section is convoluted with parton luminosity functions. The convolution scans over regions where $\beta$ is not necessarily small, unless $M$ is close to the hadronic centre-of-mass energy $s$. Hence, in these regions the threshold-enhanced terms cannot be expected a priori to give the dominant contribution to the cross section. However, one often finds after convoluting with the parton luminosity that the threshold contributions give a reasonable approximation to the total hadronic cross section (see figures 3 and 5 below for the case of squark and gluino production), so resummation is also relevant for improving predictions of the hadronic cross section.

Resummation of soft logarithms for squark and gluino production at the next-toleading (NLL) logarithmic accuracy have been presented in [14-18] using the Mellin-space resummation formalism developed by [19-22]. Recently the same formalism has been extended to NNLL order [23] and applied to squark-antisquark production [24]. These works do not resum Coulomb corrections to all orders, though the numerically dominant terms are accounted for at fixed order. All-order resummation of Coulomb contributions and bound-state effects for squark-gluino and gluino-gluino production were on the other hand investigated in [25-27], without the inclusion of soft resummation. In [28, 29] partial NNLL resummation of soft logarithms has been used to construct approximated NNLO 
results for the squark-antisquark production cross section. Recently a new formalism for the combined resummation of soft and Coulomb corrections has been developed [30, 31], and applied to NLL resummation of squark-antisquark production [31], and NNLL resummation of $t \bar{t}$ hadroproduction [32]. Contrary to the traditional Mellin-space formalism, in our approach, which is based on soft-collinear effective theory (SCET) and potential non-relativistic QCD (pNRQCD), resummation is performed directly in momentum space via renormalization-group evolution equations [33-35]. The combined soft-Coulomb effects have been found to be sizeable for the case of squark-antisquark production [31] and lead to a reduction of the scale uncertainty, as has been observed as well in [24].

In this work we extend the results given in [31] to the remaining production processes for squarks and gluinos at NLL accuracy, i.e. squark-squark, squark-gluino and gluinogluino production. We also consider separately the production of pairs of stops, which requires the extension of the formalism presented in [31] to particles pair-produced in a $P$-wave state. The paper is organized as follows: in section 2 we give an overview of squark and gluino production processes, set up the calculation and briefly review the resummation formalism we employ, listing the ingredients needed for NLL resummation. The validity of the formalism for $P$-wave induced processes, which is necessary for resummation of the stop-antistop cross section, is established in appendix A. Numerical results for the cross sections are presented in section 3, including predictions for a representative set of the benchmark points proposed in [36] and a comparison to results using the Mellinspace formalism [16, 17]. Finally in section 4 we present our conclusions and outlook. Explicit expressions for resummation functions appearing in the NLL cross sections are provided in appendix B, while appendix $\mathrm{C}$ contains some details on the scales used in the momentum-space resummation and on our method to estimate ambiguities in the resummation procedure.

\section{NLL resummation for squark and gluino production}

At hadron colliders, the dominant production channels for squarks $\tilde{q}$ and gluinos $\tilde{g}$ are pair-production processes of the form

$$
N_{1} N_{2} \rightarrow \tilde{s} \tilde{s}^{\prime} X
$$

where $N_{1,2}$ denote the incoming hadrons and $\tilde{s}, \tilde{s}^{\prime}$ the two sparticles. The total hadronic cross sections for the processes (2.1) can be obtained by convoluting short-distance production cross sections $\hat{\sigma}_{p p^{\prime}}\left(\hat{s}, \mu_{f}\right)$ for the partonic processes

$$
p p^{\prime} \rightarrow \tilde{s} \tilde{s}^{\prime} X, \quad p, p^{\prime} \in\{q, \bar{q}, g\},
$$

with the parton luminosity functions $L_{p p^{\prime}}(\tau, \mu)$ :

$$
\sigma_{N_{1} N_{2} \rightarrow \tilde{s} \tilde{s}^{\prime} X}(s)=\int_{\tau_{0}}^{1} d \tau \sum_{p, p^{\prime}=q, \bar{q}, g} L_{p p^{\prime}}\left(\tau, \mu_{f}\right) \hat{\sigma}_{p p^{\prime}}\left(\tau s, \mu_{f}\right),
$$


where $\tau_{0}=4 M^{2} / \mathrm{s}$, with the average sparticle mass

$$
M=\frac{m_{\tilde{s}}+m_{\tilde{s}^{\prime}}}{2} .
$$

The parton luminosity functions are defined from the parton density functions (PDFs) as

$$
L_{p p^{\prime}}(\tau, \mu)=\int_{0}^{1} d x_{1} d x_{2} \delta\left(x_{1} x_{2}-\tau\right) f_{p / N_{1}}\left(x_{1}, \mu\right) f_{p^{\prime} / N_{2}}\left(x_{2}, \mu\right) .
$$

We perform a NLL resummation of threshold logarithms and Coulomb corrections to the partonic cross section, counting both $\alpha_{s} / \beta$ and $\alpha_{s} \ln \beta$ as quantities of order one, where $\beta=\left(1-4 M^{2} / \hat{s}\right)^{1 / 2}$ is the heavy-particle velocity. Our predictions include all corrections to the Born cross section of the schematic form

$$
\hat{\sigma}_{p p^{\prime}}^{\mathrm{NLL}} \propto \hat{\sigma}^{(0)} \sum_{k=0}\left(\frac{\alpha_{s}}{\beta}\right)^{k} \exp \left[\ln \beta g_{0}\left(\alpha_{s} \ln \beta\right)+g_{1}\left(\alpha_{s} \ln \beta\right)\right] .
$$

A resummation at NNLL accuracy in the counting $\alpha_{s} \ln \beta \sim 1, \alpha_{s} / \beta \sim 1$, which is beyond the scope of this paper but has recently been performed for top-pair production [32], would include in addition terms of the form $\exp \left[\alpha_{s} g_{2}\left(\alpha_{s} \ln \beta\right)\right]$ and corrections of order $\mathcal{O}\left(\alpha_{s}\right)$ relative to the NLL cross section, including NLO corrections to the Coulomb potential and other higher-order potentials, as well as the non-logarithmic one-loop hard corrections. The recent NNLL calculation of squark-antisquark production [24] included the corrections of the $g_{2}$-type related to soft corrections and the hard $\mathcal{O}\left(\alpha_{s}\right)$ corrections, but kept only the $\left(\alpha_{s} / \beta\right)^{1}$-term in the sum over $k$. In section 2.1 we collect some facts about the production processes of gluinos and the superpartners of the light quarks at LO and NLO while the formalism employed for the NLL resummation is reviewed in section 2.2. The production of stop pairs is included in 2.3, while details about the choice of the soft scale in the momentum-space resummation formalism and our procedure to estimate the remaining theoretical uncertainty are discussed in 2.4.

\section{$2.1 \quad$ LO and NLO results}

At leading order [37-39], the following partonic channels contribute to the production of light-flavour squarks and gluinos:

$$
\begin{array}{rlrl}
g g, q_{i} \bar{q}_{j} & \rightarrow \tilde{q} \overline{\tilde{q}}, & & \\
q_{i} q_{j} & \rightarrow \tilde{q} \tilde{q}, & \bar{q}_{i} \bar{q}_{j} \rightarrow \overline{\tilde{q}} \overline{\tilde{q}}, \\
g q_{i} & \rightarrow \tilde{g} \tilde{q}, & g \bar{q}_{i} & \rightarrow \tilde{g} \overline{\tilde{q}}, \\
g g, q_{i} \bar{q}_{i} & \rightarrow \tilde{g} \tilde{g}, & &
\end{array}
$$

where $i, j=u, d, s, c, b$. At NLO further partonic processes contribute to the cross section. To keep the notation as simple as possible, in (2.7) we have suppressed the helicity and flavour indices of the squarks. It is understood that in the predictions for the cross sections presented below the contributions of the ten light-flavour squarks

$\left(\tilde{u}_{L / R}, \tilde{d}_{L / R}, \tilde{c}_{L / R}, \tilde{s}_{L / R}, \tilde{b}_{L / R}\right)$ are always summed over. Furthermore, the ten scalars are 

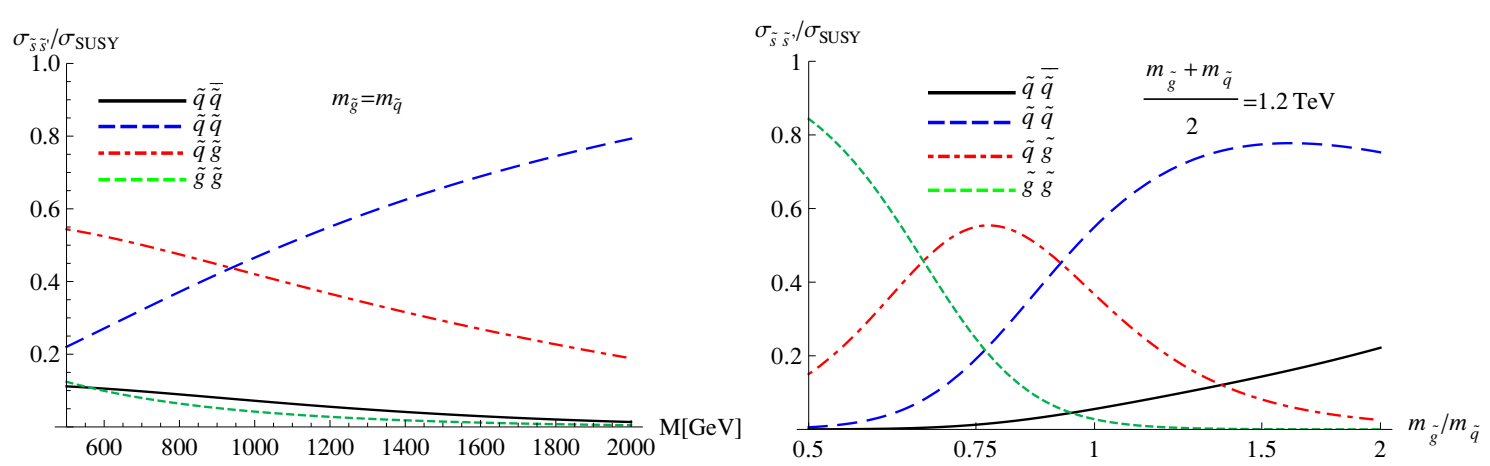

Figure 1. Ratio of the LO production cross sections for the processes (2.7) to the total Born production rate of coloured sparticles, $\sigma_{\mathrm{SUSY}}$, for the LHC with $\sqrt{s}=7 \mathrm{TeV}$. Left: Mass dependence for a fixed mass ratio $m_{\tilde{q}}=m_{\tilde{g}}=M$. Right: Dependence on the ratio $m_{\tilde{g}} / m_{\tilde{q}}$ for a fixed average $\operatorname{mass}\left(m_{\tilde{q}}+m_{\tilde{g}}\right) / 2=1.2 \mathrm{TeV}$.

assumed to be degenerate in mass, with the common light-flavour squark mass given by $m_{\tilde{q}}$. In the following, the charge-conjugate subprocesses for squark-squark and gluino-squark productions will be included in our results. As input for the convolution (2.3) we will use the MSTW08 set of PDFs [40] at the appropriate perturbative order (the LO PDFs for Born-level predictions and the NLO PDFs for the NLO and NLL results) and set the factorization scale to the average mass of the produced sparticles, $\mu_{f}=M$. We use a set of PDFs with an improved accuracy at large $x$ provided to us by the MSTW collaboration that has also been employed for the NLL results in [18].

To illustrate the relative magnitude of the various processes depending on the squark and gluino masses, the ratio of the total hadronic cross section for the processes (2.7) to the total inclusive cross section for squark and gluino production $\sigma_{\mathrm{SUSY}}=\sigma_{P P \rightarrow \tilde{q} \tilde{\tilde{q}}+\tilde{q} \tilde{q}+\tilde{g} \tilde{q}+\tilde{g} \tilde{g}}$ is shown in figure 1 for the LHC with $\sqrt{s}=7 \mathrm{TeV}$ centre-of-mass energy. From the lefthand side plot, showing the relative contributions of the various processes as a function of a common squark and gluino mass, it can be seen that squark-squark and squark-gluino production are by far the dominant channels over the full mass range considered. In the right-hand side plot, the relative contributions are shown as a function of the squarkgluino mass ratio for average mass $\frac{m_{\tilde{g}}+m_{\tilde{q}}}{2}=1.2 \mathrm{TeV}$ and it is seen that only for gluinos that are significantly lighter than squarks, gluino-pair production becomes the dominant production channel. In figure 2 we show the $K$-factor $K_{\mathrm{NLO}}=\sigma_{\mathrm{NLO}} / \sigma_{\mathrm{LO}}$ for the SUSYQCD corrections for the various production processes as obtained from PROSPINO [6]. ${ }^{1}$ The corrections are positive and enhance the cross section from $40 \%$ for squark-gluino production with light sparticle masses up to $100 \%$ or larger for squark-antisquark and gluino-pair production at large sparticle masses.

Since the focus of this work is on higher-order corrections that are enhanced in the threshold limit $\beta \rightarrow 0$, we consider here the corresponding terms appearing at NLO. To this end, we decompose the partonic cross section $\hat{\sigma}_{p p^{\prime}}$ into a complete colour basis, and

\footnotetext{
${ }^{1}$ To see the genuine size of the NLO corrections, the $K$-factors have been computed using the NLO PDFs for the Born cross sections.
} 

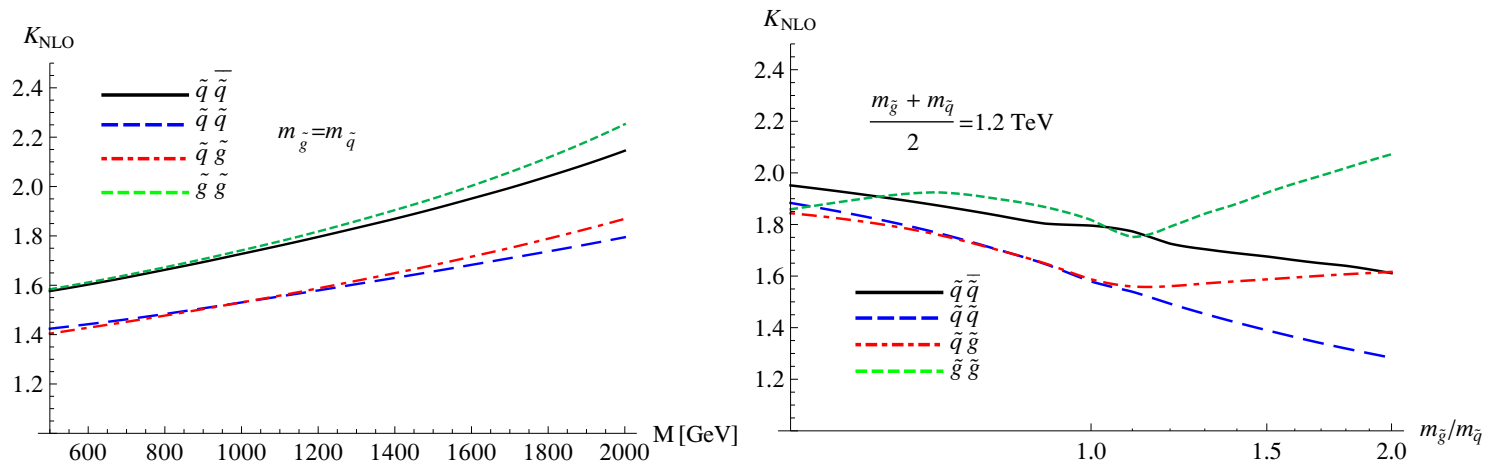

Figure 2. NLO $K$-factor for the processes (2.7) at the LHC with $\sqrt{s}=7 \mathrm{TeV}$. Left: Mass dependence for a fixed mass ratio $m_{\tilde{q}}=m_{\tilde{g}}=M$. Right: Dependence on the ratio $m_{\tilde{g}} / m_{\tilde{q}}$ for a fixed average mass $\left(m_{\tilde{q}}+m_{\tilde{g}}\right) / 2=1.2 \mathrm{TeV}$.

parametrize the higher-order corrections as

$$
\hat{\sigma}_{p p^{\prime}}=\sum_{R_{\alpha}} \hat{\sigma}_{p p^{\prime}}^{(0), R_{\alpha}}\left\{1+\frac{\alpha_{s}}{4 \pi} f_{p p^{\prime}}^{(1), R_{\alpha}}+\ldots\right\} .
$$

The sum is over the irreducible colour representations appearing in the decomposition $R \otimes R^{\prime}=\sum R_{\alpha}$, where $R, R^{\prime}$ are the $\mathrm{SU}(3)$ representations of the two final-state sparticles. The relevant decompositions for squark and gluino production are given by

$$
\begin{array}{ll}
\tilde{q} \overline{\tilde{q}}: & 3 \otimes \overline{3}=1 \oplus 8, \\
\tilde{q} \tilde{q}: & 3 \otimes 3=\overline{3} \oplus 6, \\
\tilde{q} \tilde{g}: & 3 \otimes 8=3 \oplus \overline{6} \oplus 15, \\
\tilde{g} \tilde{g}: & 8 \otimes 8=1 \oplus 8_{s} \oplus 8_{a} \oplus 10 \oplus \overline{10} \oplus 27 .
\end{array}
$$

The explicit basis tensors for the various representations have been constructed in [30] (see also $[15,16]$ ), where it has been shown that an $s$-channel colour basis based on the decompositions (2.9) is advantageous for the all-order summation of soft-gluon corrections. In eq. (2.8) $\hat{\sigma}_{p p^{\prime}}^{(0), R_{\alpha}}$ represents the tree-level cross section for a given process in colour channel $R_{\alpha}$, while the $f_{p p^{\prime}}^{(1), R_{\alpha}}$ are colour-specific NLO scaling functions. The colour-separated Born cross sections for squark and gluino production are available in [15-17]. The NLO scaling functions, on the contrary, are only known numerically in their colour-averaged form [5]. However, a simple formula is available for the threshold limit of the NLO scaling functions, containing all the threshold-enhanced contributions, for arbitrary colour representation $R_{\alpha}[41]:$

$$
\begin{aligned}
f_{p p^{\prime}}^{(1), R_{\alpha}}= & -\frac{2 \pi^{2} D_{R_{\alpha}}}{\beta} \sqrt{\frac{2 m_{r}}{M}}+4\left(C_{r}+C_{r^{\prime}}\right)\left[\ln ^{2}\left(\frac{8 M \beta^{2}}{\mu_{f}}\right)+8-\frac{11 \pi^{2}}{24}\right] \\
& -4\left(C_{R_{\alpha}}+4\left(C_{r}+C_{r^{\prime}}\right)\right) \ln \left(\frac{8 M \beta^{2}}{\mu_{f}}\right)+12 C_{R_{\alpha}}+h_{p p^{\prime}}^{(1), R_{\alpha}}+\mathcal{O}(\beta)
\end{aligned}
$$

with $M$ the average mass of the two particles produced (2.4), while $m_{r}$ denotes the reduced mass, $m_{r}=m_{\tilde{s}} m_{\tilde{s}^{\prime}} /\left(m_{\tilde{s}}+m_{\tilde{s}^{\prime}}\right) . C_{r}, C_{r^{\prime}}$ and $C_{R_{\alpha}}$ are the Casimir invariants for the colour 


\begin{tabular}{|c|c|c|c|l|}
\hline$\tilde{q} \tilde{\tilde{q}}$ & $D_{1}=-\frac{4}{3}$ & $D_{8}=\frac{1}{6}$ & & \\
\hline$\tilde{q} \tilde{q}$ & $D_{\overline{3}}=-\frac{2}{3}$ & $D_{6}=\frac{1}{3}$ & & \\
\hline$\tilde{g} \tilde{q}$ & $D_{3}=-\frac{3}{2}$ & $D_{\overline{6}}=-\frac{1}{2}$, & $D_{15}=+\frac{1}{2}$ & \\
\hline$\tilde{g} \tilde{g}$ & $D_{1}=-3$ & $D_{8}=-\frac{3}{2}$ & $D_{10}=0$ & $D_{27}=1$ \\
\hline
\end{tabular}

Table 1. Numerical values of the coefficients of the Coulomb potential (2.11) for squark and gluino production processes. Negative values correspond to an attractive potential.

representations of the initial-state particles, $p$ and $p^{\prime}$, and for the irreducible representation $R_{\alpha}$ of the SUSY pair. The coefficients $D_{R_{\alpha}}$ of the Coulomb potential for the production of heavy particles in $\mathrm{SU}(3)$ representations $R$ and $R^{\prime}$ in the colour channel $R_{\alpha}$ are given in terms of the quadratic Casimir operators for the various representations:

$$
D_{R_{\alpha}}=\frac{1}{2}\left(C_{R_{\alpha}}-C_{R}-C_{R^{\prime}}\right)
$$

where negative values correspond to an attractive Coulomb potential, positive values to a repulsive one. The numerical values for the representations relevant for squark and gluino production can be found in $[31,42]$ and are collected in table 1 . The coefficient $h_{p p^{\prime}}^{(1), R_{\alpha}}$ is the one-loop contribution to the hard matching coefficient appearing in eq. (2.13) below, and represents the only process-specific quantity in eq. (2.10). It has been obtained recently for squark-antisquark production and gluino-gluino production $[24,26]$ but is not known yet for the remaining production processes. The knowledge of $h_{p p^{\prime}}^{(1), R_{\alpha}}$ is required for NNLL resummation [24], but not at NLL accuracy as considered here, so the $h_{p p^{\prime}}^{(1), R_{\alpha}}$ will be always set to zero in the following. Using the Born cross sections for the different colour channels $[15,16]$ and $(2.10)$ one can reproduce the threshold expansions of the NLO corrections in $[5] .^{2}$

In figure 3 we study to which extent the full NLO corrections as obtained from PROSPINO are approximated by the singular NLO corrections, obtained by dropping all constant terms from (2.10), including $\ln 2$ terms, and convoluting the resulting partonic cross section (2.8) with the parton luminosities. For the Born cross sections $\hat{\sigma}_{p p^{\prime}}^{(0), R_{\alpha}}$ in (2.8) the exact expressions, without use of the threshold approximation, have been kept, but colour channels with a vanishing threshold limit of the Born cross section at leading order in $\beta$ have been dropped. For the case of degenerate squark and gluino masses it is seen that the difference of the threshold-enhanced contributions to the full NLO corrections is at the 10-30\% level over the whole mass range considered, with the exception of the squark-squark production channel where the threshold contributions account for only

\footnotetext{
${ }^{2}$ In [5] there is a typo in the sign of the Coulomb correction for like-flavour $\tilde{q} \tilde{q}$ production, i.e. the function $f_{q q}^{V+S}$ in eq. (54). Also note that [5] choose to expand the cross section of $\tilde{q} \tilde{g}$ production in the variable $\bar{\beta}=\sqrt{1-4 m_{\tilde{q}} m_{\tilde{g}} /\left(s-\left(m_{\tilde{q}}-m_{\tilde{g}}\right)^{2}\right)} \approx \beta \sqrt{M / 2 m_{r}}$ which leads to the appearance of additional $\ln \left(m_{r} / M\right)$ terms. For this process they also observe an apparent non-factorization of the colour-averaged NLO threshold corrections from the Born cross section. This is nevertheless consistent with (2.10) since the Born $\tilde{q} \tilde{g}$ cross section in the colour-triplet channel is not proportional to that for the other channels [16].
} 

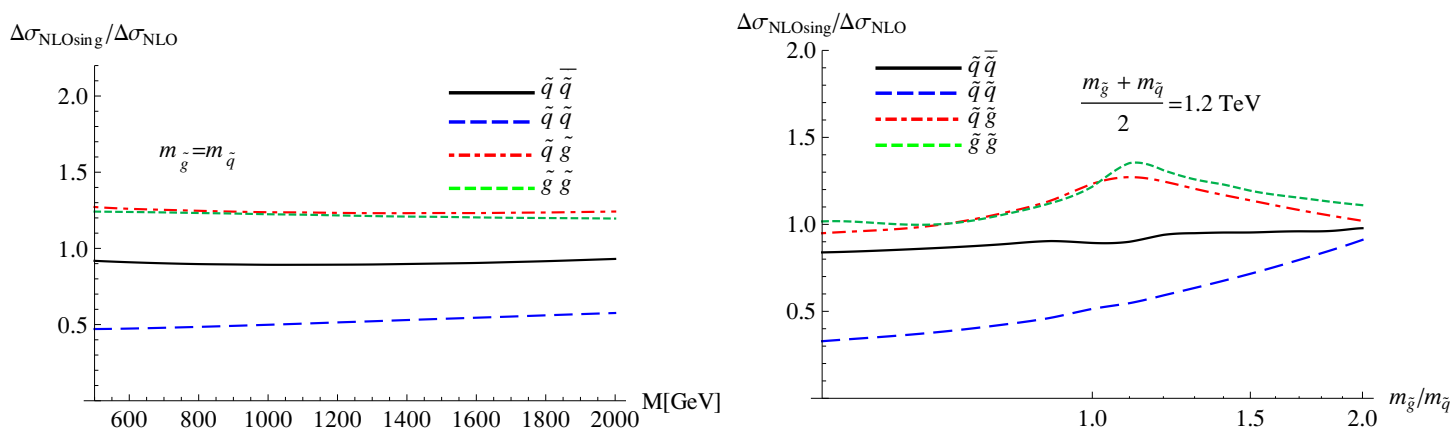

Figure 3. Ratio of the singular NLO contributions obtained from (2.10) to the exact NLO corrections for the LHC with $\sqrt{s}=7 \mathrm{TeV}$. Left: Mass dependence for a fixed mass-ratio $m_{\tilde{q}}=m_{\tilde{g}}=M$. Right: Dependence on the ratio $m_{\tilde{g}} / m_{\tilde{q}}$ for a fixed average mass $\left(m_{\tilde{q}}+m_{\tilde{g}}\right) / 2=1.2 \mathrm{TeV}$.

$40-50 \%$ of the full NLO corrections. For $m_{\tilde{g}}>m_{\tilde{q}}$ the singular terms overestimate the corrections for the processes involving gluinos, while the agreement for squark-antisquark production improves. For $m_{\tilde{g}}<m_{\tilde{q}}$ the singular terms approximate the full corrections very well for all processes apart from squark-squark production. ${ }^{3}$ Comparing to figure 1 , it is seen that the singular terms capture the NLO corrections to the dominant processes for larger mass ratios (i.e. squark-squark production for $m_{\tilde{g}} \sim 2 m_{\tilde{q}}$ and gluino-pair production for $m_{\tilde{g}} \sim 0.5 m_{\tilde{q}}$ ) rather well. For degenerate squark and gluino masses the quality of the threshold approximation for the dominant squark-squark and squark-gluino processes is somewhat worse. In all cases, the inclusion of the threshold enhanced NLO corrections in addition to the Born terms improves the agreement with the full NLO results. This motivates the computation of the higher-order threshold-enhanced terms through resummation, as performed in the remainder of this work.

\subsection{Soft-gluon and Coulomb resummation}

Next, we briefly review the formalism for the combined resummation of soft- and Coulombgluon corrections [30,31] and provide the relevant ingredients for squark and gluino production at NLL accuracy. We also discuss some features of our implementation that differ from that used previously for squark-antisquark production in [31].

The combined soft-Coulomb resummation for the production of squarks and gluinos is based on a factorization of the hard-scattering total cross section for partonic subprocesses of the type (2.2). It can be shown that near the partonic threshold,

$$
\hat{s} \sim\left(m_{\tilde{s}}+m_{\tilde{s}^{\prime}}\right)^{2},
$$

the partonic cross section factorizes into three contributions [31], a hard function $H$, a soft function $W$ containing soft gluons to all orders, and a potential function $J$ summing

\footnotetext{
${ }^{3}$ Note that one could improve the threshold approximation by including the constant terms in (2.10) once the coefficients $h_{p p^{\prime}}^{(1), R_{\alpha}}$ are known. This could be particularly relevant for the squark-squark process where the threshold contributions to the NLO cross section are relatively small due to the smaller colour charges involved and an accidental cancellation of Coulomb corrections between the same-flavour and differentflavour production channels.
} 
Coulomb-gluon exchange:

$$
\hat{\sigma}_{p p^{\prime}}(\hat{s}, \mu)=\sum_{R_{\alpha}} H_{p p^{\prime}}^{R_{\alpha}}\left(m_{\tilde{q}}, m_{\tilde{g}}, \mu\right) \int d \omega J_{R_{\alpha}}\left(E-\frac{\omega}{2}\right) W^{R_{\alpha}}(\omega, \mu) .
$$

Here $E=\sqrt{\hat{s}}-2 M$ is the energy relative to the production threshold and the sum is over the colour representations (2.9). In (2.13) the $s$-channel colour basis mentioned above, that can be shown to diagonalize the soft function to all orders [30], is chosen for the hard-scattering amplitudes. Independent of the sparticle type, the soft function then depends only on the colour representations of the initial-state partons and the irreducible representation $R_{\alpha}$ of the sparticle pair appearing in the decompositions (2.9), in agreement with the picture that soft-gluon radiation is only sensitive to the total colour charge of the slowly moving sparticle pair [22]. The formula (2.13) has been derived in [31] for $S$-wave dominated production processes up to NNLL accuracy. This covers all production processes of squarks and gluinos, apart from quark-antiquark initiated stop-antistop production, that proceeds through a $P$-wave. The applicability of the formalism to stop production is discussed in section 2.3 and appendix A.

It can be argued that the natural scale for the evaluation of the hard function in (2.13), leading to well-behaved higher-order corrections, is of the order of $\mu_{h} \sim 2 M$, while the natural scale for soft-gluon radiation is of the order of $\mu_{s} \sim M \beta^{2}$. We use the momentumspace resummation formalism of [33-35] to evolve the soft and hard functions from their natural scales to the factorization scale $\mu_{f}$ used for the evaluation of the parton distribution functions, commonly taken to be of the order of $\mu_{f} \sim M$. In this way, logarithms of $\mu_{s} / \mu_{f} \sim \beta^{2}$ are summed to all orders. The precise prescription for the choice of the soft scale adopted in our calculation is discussed in 2.4. The exchange of multiple Coulomb gluons can be summed up using the method of Coulomb Green's functions in non-relativistic QCD [43-45].

For resummation at NLL accuracy, the leading-order hard and soft functions are required as fixed-order input to the evolution equations. The leading-order soft function is trivial, $W^{(0) R_{\alpha}}(\omega)=\delta(\omega)$. The leading-order hard functions are obtained from the threshold limit of the Born cross section for the colour channel $R_{\alpha}$ [31],

$$
\hat{\sigma}_{p p^{\prime}}^{(0), R_{\alpha}}(\hat{s}) \underset{\hat{s} \rightarrow 4 M^{2}}{\approx} \frac{\left(2 m_{r}\right)^{2}}{2 \pi} \sqrt{\frac{E}{2 m_{r}}} H_{p p^{\prime}}^{(0), R_{\alpha}} .
$$

Although the Born-cross sections in the threshold limit appear on the left-hand side in (2.14), we keep the exact expressions in our numerical implementation, so the hard functions in practice depend on $\hat{s}$. This incorporates some higher-order terms in $\beta$, albeit not systematically. ${ }^{4}$ Here our current treatment differs from that used for squark-antisquark production in [31] where only the threshold limit of the Born cross section was used to compute the hard function.

\footnotetext{
${ }^{4}$ This corresponds to the treatment of [15-17], up to the fact that we set the hard function for a given production and colour channel to zero if the Born cross section vanishes at threshold at leading order in $\beta$, even if the full Born cross section for this channel is non-vanishing. This affects the subprocesses $q \bar{q} \rightarrow \tilde{g} \tilde{g}$ in the singlet channel and $q_{i} q_{i} \rightarrow \tilde{q}_{i} \tilde{q}_{i}$ in the triplet channel. The numerical effect is however negligible.
} 
For the resummation of Coulomb corrections, we use results for the non-relativistic Coulomb Green's function obtained for top-quark production at electron-positron colliders and stop production [43, 46]. For positive values of $E$ and vanishing decay widths of the sparticles, the $S$-wave potential function is given by the Sommerfeld factor

$$
J_{R_{\alpha}}(E)=\frac{\left(2 m_{r}\right)^{2} \pi D_{R_{\alpha}} \alpha_{s}}{2 \pi}\left(e^{\pi D_{R_{\alpha}} \alpha_{s} \sqrt{\frac{2 m_{r}}{E}}}-1\right)^{-1}, \quad E>0
$$

with the coefficients $D_{R_{\alpha}}$ of the Coulomb potential given in (2.11). For an attractive potential, a series of bound states develops below threshold with energies

$$
E_{n}=-\frac{2 m_{\mathrm{r}} \alpha_{s}^{2} D_{R_{\alpha}}^{2}}{4 n^{2}}
$$

Their contribution to the $S$-wave potential function is given by

$$
J_{R_{\alpha}}^{\text {bound }}(E)=2 \sum_{n=1}^{\infty} \delta\left(E-E_{n}\right)\left(\frac{2 m_{\mathrm{r}}\left(-D_{R_{\alpha}}\right) \alpha_{s}}{2 n}\right)^{3} \quad E<0 \text {. }
$$

For sufficiently broad squarks and gluinos with decay widths exceeding the binding energy of the would-be bound states, $\Gamma_{\tilde{s}}+\Gamma_{\tilde{s}^{\prime}}>\left|E_{1}-E_{2}\right|$, the bound state poles are smeared out by the finite lifetimes. ${ }^{5}$ We consider here a situation where the widths of the squarks and gluinos are large enough to prevent the formation of bound states, but small enough that the use of a narrow-width approximation is justified, which is the case for SUSY scenarios with moderate mass ratios of squarks and gluinos where $\Gamma_{\tilde{s}} / M_{\tilde{s}} \sim 1 \%$. The contributions to the total cross section below the nominal production threshold, $\hat{s}=2 M$, can then be included by setting the sparticle widths to zero and including the bound-state poles (2.17). For other observables, the finite width can be taken into account to a first approximation by the replacement $E \rightarrow E+i\left(\Gamma_{\tilde{s}}+\Gamma_{\tilde{s}^{\prime}}\right) / 2$ in the potential function, see e.g. [25-27] for recent studies of the invariant-mass spectrum of gluino-pair and squark-gluino production. The study of finite-width corrections for larger decay widths (e.g. for gluino masses $m_{\tilde{g}} \gtrsim 2 m_{\tilde{q}}$ ) is left for future work. In the numerical results presented in this work, the contributions of the bound state poles for $E<0$ will always be included in our default implementation, and are convoluted with the resummed soft function as described in [32]. Note that in the previous results for squark-antisquark production [31] the bound-state corrections have been added without soft-gluon resummation.

The resummed cross section at NLL accuracy is obtained by inserting the potential function (2.15) and the solutions to the evolution equations of the hard and soft functions $[30,49]$ into the factorization formula (2.13). Using the solutions in momentum-space

\footnotetext{
${ }^{5}$ The actual formation of bound states is possible if the decay widths of the sparticles are smaller than the decay-rate of the bound state. For long-lived gluinos, or light stops without allowed two-body decays, higher-order corrections to bound-state production have been recently obtained in $[47,48]$. Since the bound states decay into gluon or photon pairs, this scenario leads to very different collider signatures compared to the missing-energy signatures of continuum production and is not considered further here.
} 
obtained in [35], the NLL cross section is written as

$$
\hat{\sigma}_{p p^{\prime}}^{\mathrm{NLL}}\left(\hat{s}, \mu_{f}\right)=\sum_{R_{\alpha}} H_{p p^{\prime}}^{(0), R_{\alpha}}\left(\mu_{h}\right) U_{i}\left(M, \mu_{h}, \mu_{s}, \mu_{f}\right) \frac{e^{-2 \gamma_{E} \eta}}{\Gamma(2 \eta)} \int_{0}^{\infty} d \omega \frac{J_{R_{\alpha}}\left(M \beta^{2}-\frac{\omega}{2}\right)}{\omega}\left(\frac{\omega}{2 M}\right)^{2 \eta} .
$$

Here the label $i$ jointly refers to the colour of the initial-state partons and the representation $R_{\alpha}$ of the sparticle pair. The function $\eta=\frac{2 \alpha_{s}\left(C_{r}+C_{r^{\prime}}\right)}{\pi} \ln \left(\mu_{s} / \mu_{f}\right)+\ldots$ contains single logarithms, while the resummation function $U_{i}$ sums the Sudakov double logarithms $\alpha_{s} \log ^{2} \frac{\mu_{h}}{\mu_{f}}$ and $\alpha_{s} \log ^{2} \frac{\mu_{s}}{\mu_{f}}$. Explicit expressions up to NLL accuracy are given in appendix B. For $\mu_{s}<\mu_{f}$ the function $\eta$ is negative and the factor $\omega^{2 \eta-1}$ in the resummed cross section (2.18) has to be understood in the distributional sense, as discussed in detail in [32]. We have used the non-relativistic expression $E=M \beta^{2}$ in the argument of the potential function, that is valid near the partonic threshold (2.12). This follows the default treatment of top-pair production in [32] and leads to the customary expansion of the cross section in $\beta$ (see eq. 2.10). We also perform this replacement in the definition of the hard functions (2.14). The difference between this default implementation and the results obtained by consistently keeping the expression $E=\sqrt{\hat{s}}-2 M$ (as in the previous results for squark-antisquark production [31]) will be used to estimate the effect of subleading terms in the cross section, as discussed in section 2.4.

In order to assess the importance of the Coulomb corrections and to compare to the results of the Mellin approach [16] we will also present results without Coulomb resummation, obtained by inserting the trivial potential function $J^{(0)}(E)=\frac{\left(2 m_{r}\right)^{2}}{2 \pi} \sqrt{E / 2 m_{r}}$ into (2.18). In this approximation, that we will denote by $\mathrm{NLL}_{s+h}$, a fully analytical expression for the resummed cross section can be obtained:

$$
\hat{\sigma}_{p p^{\prime}}^{\mathrm{NLL}_{s+h}}=\sum_{R_{\alpha}} \hat{\sigma}_{p p^{\prime}}^{(0)}\left(\hat{s}, \mu_{h}\right) U_{i}\left(M, \mu_{h}, \mu_{s}, \mu_{f}\right) \frac{\sqrt{\pi} e^{-2 \eta \gamma_{E}}}{2 \Gamma\left(2 \eta+\frac{3}{2}\right)} \beta^{4 \eta} .
$$

Since contributions to the cross section from outside the threshold region can be numerically non-negligible, we match the NLL resummed cross section to the fixed-order NLO calculation by subtracting the NLO expansion of the NLL expression and adding back the full NLO corrections:

$$
\hat{\sigma}_{p p^{\prime}}^{\operatorname{matched}}(\hat{s})=\left[\hat{\sigma}_{p p^{\prime}}^{\mathrm{NLL}}(\hat{s})-\hat{\sigma}_{p p^{\prime}}^{\mathrm{NLL}(1)}(\hat{s})\right]+\hat{\sigma}_{p p^{\prime}}^{\mathrm{NLO}}(\hat{s}),
$$

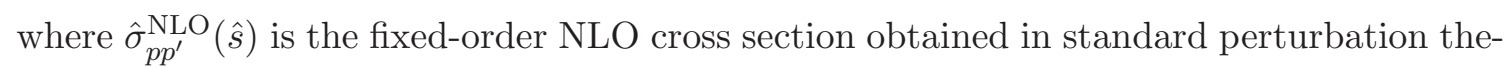
ory, as implemented in PROSPINO [6], and $\hat{\sigma}_{p p^{\prime}}^{\mathrm{NLL}(1)}$ is the resummed cross section expanded to NLO, as given in [31]. The total hadronic cross section at NLL is then obtained by convoluting (2.20) with the parton luminosity, as in (2.3).

\subsection{Stop-antistop production}

Beside the channels listed in (2.7), in section 3 we will also present predictions for stop-pair production:

$$
g g, q_{i} \bar{q}_{i} \rightarrow \tilde{t}_{j} \overline{\tilde{t}}_{j}
$$




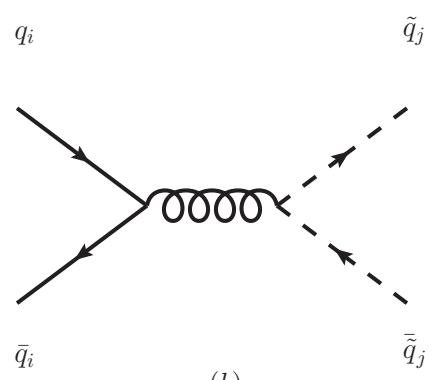

(a)

$(b)$

Figure 4. Tree-level diagram topologies contributing to $q_{k} \bar{q}_{l} \rightarrow \tilde{q}_{i} \overline{\tilde{q}}_{j}$.
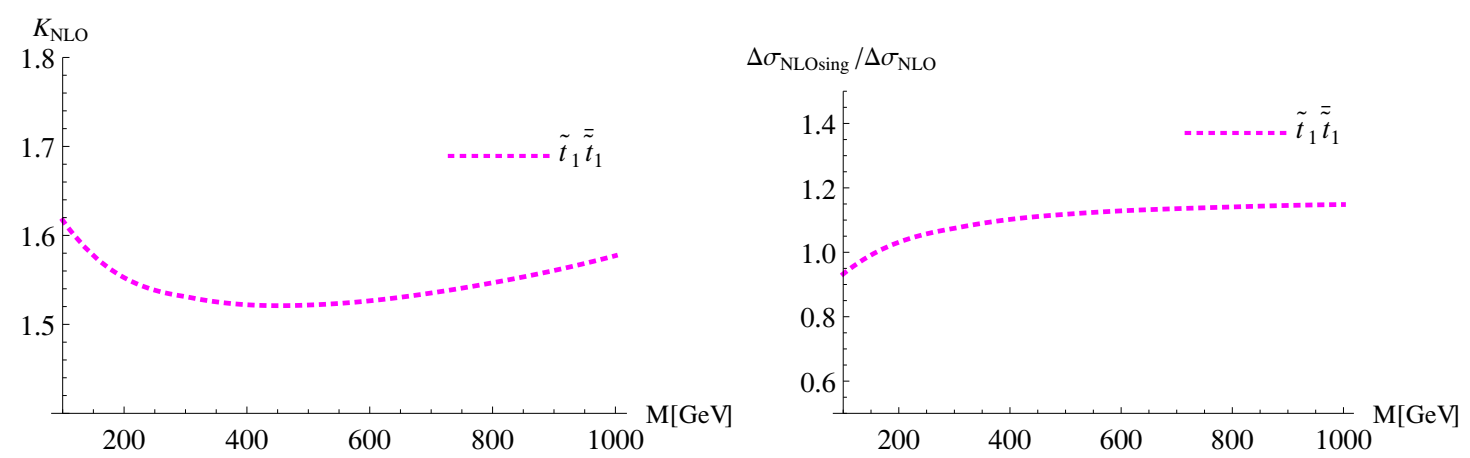

Figure 5. Left: NLO $K$-factor for stop-pair production at $\sqrt{s}=7 \mathrm{TeV}$ as a function of the stop mass. Right: ratio of the singular NLO contributions obtained from eqs. (2.10) and (2.25) to the full NLO cross section for $P P \rightarrow \tilde{t}_{1} \overline{\tilde{t}}_{1}$.

where only the initial states appearing at leading order have been shown. The NLO SUSYQCD corrections have been computed in [7] and are implemented in PROSPINO [6]. Contrary to the light-flavour squark case, in most scenarios the mixing of the two weak eigenstates $\tilde{t}_{L}, \tilde{t}_{R}$, and the mass difference of the resulting mass eigenstates, $\tilde{t}_{1}=\tilde{t}_{L} \cos \theta_{\tilde{t}}+\tilde{t}_{R} \sin \theta_{\tilde{t}}$, $\tilde{t}_{2}=-\tilde{t}_{L} \sin \theta_{\tilde{t}}+\tilde{t}_{R} \cos \theta_{\tilde{t}}$, is non-negligible. Off-diagonal production of the mass eigenstates, e.g. $\tilde{t}_{1} \tilde{t_{2}}$, appears at NLO in SUSY-QCD, and through electroweak contributions. It is therefore suppressed compared to diagonal production $[7,50]$ and will not be considered here. It must also be mentioned that because of the absence of a significant top-quark component inside the nucleon the processes $\tilde{t} \tilde{t}$ and $\tilde{g} \tilde{t}$ first contribute to the cross section at NLO, and are thus numerically suppressed. The NLO $K$-factor for the process $P P \rightarrow \tilde{t}_{1} \overline{\tilde{t}}_{1}$ is shown in figure 5 for a centre-of-mass energy of $7 \mathrm{TeV}$, and the mass range $M=100$ $1000 \mathrm{GeV}$. As can be seen, NLO corrections are in the $50-60 \%$ range. The predictions for the second mass eigenstate differ only in the fixed-order NLO results, and for a given mass the numerical difference between the cross sections for $\tilde{t}_{1} \overline{\tilde{t}}_{1}$ and $\tilde{t}_{2} \overline{\tilde{t}}_{2}$ production is below $2 \%$ for the mass range considered in this work. We therefore omit results for the process $P P \rightarrow \tilde{t}_{2} \overline{\tilde{t}}_{2}$.

Contrary to the production of a light squark-antisquark pair, stop-pair production in the $q \bar{q}$ channel cannot be mediated by a $t$-channel diagram like in figure 4(a), again due to the extreme suppression of top-quark PDFs inside the proton. As a result, at LO in QCD 
a stop-antistop pair is produced in a $P$-wave state in quark-antiquark collisions. As shown in appendix A, the resummation formalism can be extended in a straightforward way to $q \bar{q}$-initiated stop-antistop production at NLL, and the only modification of (2.13) is the replacement of the potential function by that appropriate for $P$-wave processes. For stable particles, above threshold the result is given by [46] (see also [51])

$$
J_{R_{\alpha}}^{P}(E)=2 m_{r} E\left(1+\frac{\left(\alpha_{s} D_{R_{\alpha}}\right)^{2} 2 m_{r}}{4 E}\right) J_{R_{\alpha}}(E), \quad E>0 .
$$

The bound-state contributions of the $P$-wave Green's function can be found in [46] but are not needed here, since only the repulsive colour-octet channel appears in our application to stop-pair production. By expanding (2.22) in the strong coupling constant one obtains the coefficients of the fixed-order Coulomb corrections. While the one-loop Coulomb correction agrees with that for $S$-wave production (2.10), the second Coulomb correction $\frac{\left(D_{R_{\alpha}} \alpha_{s}\right)^{2}}{12}\left(\pi^{2}+3\right) / \beta^{2}$ differs from the $S$-wave case. Due to the different normalization of the $P$-wave Green's function, the definition of the leading-order hard functions for $P$-wave production reads

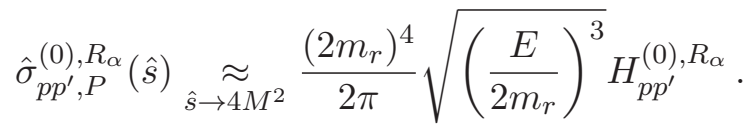

In addition to the the combined soft/Coulomb resummation, we will again consider the $\mathrm{NLL}_{s+h}$ approximation where the trivial $P$-wave potential function $J^{(0)}(E)=$ $\frac{\left(2 m_{r}\right)^{4}}{2 \pi}\left(E / 2 m_{r}\right)^{3 / 2}$ is used in the resummation formula, leading to the analytical result

$$
\hat{\sigma}_{p p^{\prime}, P}^{\mathrm{NLL}_{s+h}}=\sum_{R_{\alpha}} \hat{\sigma}_{p p^{\prime}}^{(0)}\left(\hat{s}, \mu_{h}\right) U_{i}\left(M, \mu_{h}, \mu_{s}, \mu_{f}\right) \frac{3 \sqrt{\pi} e^{-2 \eta \gamma_{E}}}{4 \Gamma\left(2 \eta+\frac{5}{2}\right)} \beta^{4 \eta} .
$$

In analogy to the $S$-wave result (2.10), one can use the resummation formalism to obtain the threshold-enhanced one-loop scaling functions for $P$-wave production in the colour channel $R_{\alpha}$ from initial-state partons in the representations $r$ and $r^{\prime}$ :

$$
\begin{aligned}
f_{p p^{\prime}, P}^{(1) R_{\alpha}}=-\frac{2 \pi^{2} D_{R_{\alpha}}}{\beta} & \sqrt{\frac{2 m_{r}}{M}}+4\left(C_{r}+C_{r^{\prime}}\right)\left[\ln ^{2}\left(\frac{8 E}{\mu_{f}}\right)+\frac{104}{9}-\frac{11 \pi^{2}}{24}\right] \\
& -4\left(C_{R_{\alpha}}+\frac{16}{3}\left(C_{r}+C_{r^{\prime}}\right)\right) \ln \left(\frac{8 E}{\mu_{f}}\right)+\frac{44}{3} C_{R_{\alpha}}+h_{i}^{(1)}\left(\mu_{f}\right) .
\end{aligned}
$$

In agreement with [17] one finds that the coefficient of the single logarithms related to initial-state radiation is multiplied by a factor of $\frac{4}{3}$ compared to the $S$-wave case while the double logarithm and the logarithms related to final state radiation proportional to $C_{R_{\alpha}}$ are unchanged. In addition, the constant terms are different which is irrelevant at NLL accuracy but has to be taken into account if one aims to extract the one-loop hard function from a computation of the NLO cross section. In figure 5 we study the accuracy of the threshold approximation defined by inserting the NLO singular terms, obtained from eqs. (2.10) and (2.25) by dropping constant terms, into (2.8). The ratio of the singular NLO corrections to the full NLO corrections to the hadronic cross section obtained using PROSPINO is shown in figure 5 (right plot). Analogously to squark-antisquark production, the threshold terms provide an excellent approximation of the full NLO result. 


\subsection{Scale choices}

As explained in section 2.2, the resummed partonic cross section (2.13) depends on a number of scales related to the factorization of hard, soft and Coulomb effects. The dependence on these scales would cancel in the exact result, but a residual dependence remains at a given logarithmic order. As already pointed out, our default choice for the factorization and hard scales are $\mu_{f} \equiv M$ and $\mu_{h} \equiv 2 M$, respectively. On the other hand, the resummation of all NLL effects related to Coulomb exchange requires that the scale in the potential function $J_{R_{\alpha}}$ is chosen of the order of $\sqrt{2 m_{r} M} \beta$, which is the typical virtuality of Coulomb gluons. A more detailed analysis shows in fact that for an attractive Coulomb potential the Coulomb scale freezes when $\beta \sim\left|D_{R_{\alpha}}\right| \alpha_{s}$, due to bound-state formation. We thus choose the scale in $J_{R_{\alpha}}$ to be

$$
\mu_{C}=\operatorname{Max}\left\{2 \alpha_{s}\left(\mu_{C}\right) m_{r}\left|D_{R_{\alpha}}\right|, 2 \sqrt{2 m_{r} M} \beta\right\} .
$$

Note that, for a repulsive potential, $D_{R_{\alpha}}>0$, no bound states arise, so that $(2.26)$ is not completely justified. However in this case resummation of Coulomb corrections leads to small effects, and $J_{R_{\alpha}}$ vanishes for small $\beta$, so that the precise choice of $\mu_{C}$ in this limit has a negligible numerical impact on predictions of the cross section.

The choice of the soft scale $\mu_{s}$ presents some subtleties. The exponentiation of all NLL $\ln \beta$-terms in the partonic cross section would require a choice $\mu_{s} \sim M \beta^{2}$. However a running scale leads to strong oscillations of the cross section for small $\beta$, due to the prefactor $e^{-2 \gamma_{E} \eta} / \Gamma(2 \eta)$ in (2.18), amplified by the factor $\omega^{2 \eta}$ and terms in the function $U_{i}$, and eventually hits the Landau pole of the strong coupling constant $\alpha_{s}$ when $\beta \rightarrow 0$. To overcome these problems two different approaches have been used in the literature:

Fixed $\mu_{s}:$ in [33-35] the choice of a fixed soft scale was advocated. Such a scale is determined from the minimization of the one-loop soft corrections to the hadronic cross section,

$$
0=\mu_{s} \frac{d}{d \mu_{s}} \sum_{p, p^{\prime}} \int_{\tau_{0}}^{1} d \tau L_{p p^{\prime}}\left(\tau, \mu_{s}\right) \frac{\hat{\sigma}_{p p^{\prime}, \text { soft }}^{(1)}\left(\tau s, \mu_{s}\right)}{\sigma_{N_{1} N_{2}}^{(0)}\left(s, \mu_{s}\right)} .
$$

In this approach threshold logarithms are resummed in an average sense and not locally at the level of the partonic cross section. However one can argue that, for threshold dominated processes, the choice (2.27) preserves the hierarchy between the soft and shortdistance scales and that $\operatorname{logarithmic}$ corrections $\log \left(1-\tau_{0}\right)$ to the hadronic cross section are correctly resummed. This was the method adopted in [31] for resummation of the squark-antisquark production cross section. The explicit value of the scales determined with the minimization procedure (2.27) are given in eq. (C.1) in appendix C.

Running $\boldsymbol{\mu}_{\boldsymbol{s}}$ : for the NNLL resummation of soft effects in $t \bar{t}$ production presented in [32] a different approach was adopted. There a running soft scale,

$$
\mu_{s}^{>}=k_{s} M \beta^{2},
$$

was used in the interval $\beta>\beta_{\text {cut }}$, and replaced by a fixed soft scale

$$
\mu_{s}^{<}=k_{s} M \beta_{\text {cut }}^{2}
$$


below the cutoff. With this scale choice, logarithms of $\beta$ are exponentiated locally in the partonic cross section in the large- $\beta$ region, where the use of a fixed soft scale cannot be a priori justified. On the other hand if $\beta_{\text {cut }}$ is not too big, in the lower interval the hadronic cross section is in fact dominated by logarithms of $\beta_{\text {cut }}$, as can be explicitly checked by convoluting the partonic cross section with toy parton luminosities [35], so that the use of a fixed scale once again correctly resums the dominant logarithms. The precise value of $\beta_{\text {cut }}$ is chosen through the prescription described in [32], which is reviewed in appendix C. The default choice for the prefactor $k_{s}$ adopted here is $k_{s}=1$. We have observed that, for the SUSY processes considered here, the NLL expression and its NNLO expansion are generally stable against variations of $k_{s}$ for this choice. ${ }^{6}$

The two possible choices of the soft scale $\mu_{s}$ just discussed are one of the ambiguities associated with threshold resummation. Others are related to the choice of the hard and Coulomb scales and to power-suppressed terms which are not controlled by resummation. Additionally, one has to consider the ambiguity arising from the choice of the factorization scale $\mu_{f}$. The latter clearly also applies to the fixed-order NLO result. Thus, to reliably ascertain the residual uncertainty of the fixed-order and resummed results we present in section 3, we adopt the following procedure:

- Scale uncertainty: for both the NLO and NLL result the factorization scale $\mu_{f}$ is varied between half and twice the default value, i.e. $M / 2<\mu_{f}<2 M$. For the NLL result, this is done keeping the other scales $\mu_{h}, \mu_{C} \mu_{s}$ and the parameters $\beta_{\text {cut }}$ and $k_{s}$ fixed.

- Resummation uncertainty: both hard and Coulomb scales are varied between half and twice the default values, i.e. $M<\mu_{h}<4 M$ and $\mu_{C}^{(0)} / 2<\mu_{C}<2 \mu_{C}^{(0)}$, where $\mu_{C}^{(0)}$ is the solution of the implicit equation (2.26). In addition, for the NLL implementation with a fixed soft scale, $\mu_{s}$ is varied between half and twice its default value, while for the running-scale implementation uncertainties related to the choice of $\beta_{\text {cut }}$ and $k_{s}$ are estimated according to the procedure given in [32] (and reviewed in appendix C). Finally, as anticipated below (2.18), we take the difference in parametrizing the resummed cross section in terms of $\beta$ or $\hat{E}$ as a measure of the effect of power-suppressed terms. All the scales and the parameters $\beta_{\text {cut }}$ and $k_{s}$ are varied one at the time keeping the other fixed to their central values, and the resulting errors are summed in quadrature.

- PDF uncertainty: we estimate the error due to uncertainties in the PDFs using the $68 \%$ confidence level eigenvector set of the MSTW08NLO PDFs [40].

An additional source of error arises from the uncertainty on the $\alpha_{s}$-determination. This effect has been found to be of the order of $3 \%$ for the NLO cross sections of squarksquark, squark-antisquark and squark gluino production and up to $8 \%$ for gluino-pair production [18]. We expect a similar uncertainty of the NLL results.

\footnotetext{
${ }^{6}$ Our choice of $k_{s}$ deviates from the one adopted in [32], where $k_{s}=2$. This corresponds to resumming some of the $\ln 2$ terms in the fixed-order cross section alongside the threshold-enhanced $\ln \beta$ contributions.
} 
In the following we will often refer to the sum in quadrature of scale and resummation uncertainty as "total theoretical uncertainty". Note that the terminology adopted here differs slightly from the one used for $t \bar{t}$ production in [32] where the errors from variation of the hard and Coulomb scales, and of the soft scale for the fixed-scale implementation, had been incorporated into the scale uncertainty, while we consider them as resummation ambiguities. Additionally, in [32] independent and simultaneous variations of the factorization and renormalization scale have been considered, whereas in this work we identify the factorization and renormalization scales and vary them as one scale, i.e. $M / 2<\mu_{f} \equiv \mu_{r}<2 M$. This is the default procedure implemented in the numerical code PROSPINO used for the computation of the fixed-order NLO result [6].

It is interesting to study how the choice of a fixed or running soft scale affects the NLL resummed cross section, especially in view of the uncertainties just discussed. In figure 6 we plot the NLL $K$-factor, defined in eq. (3.2), as a function of a common SUSY mass $M=m_{\tilde{g}}=m_{\tilde{q}}$ for the four processes listed in (2.7), for a centre-of-mass energy of $7 \mathrm{TeV}$ (the situation for $\sqrt{s}=14 \mathrm{TeV}$ is qualitatively similar). Results for the stop-pair production process and the total SUSY production rate are also shown. The thick lines represent the central values for the two implementations, whereas the bands (delimited by thinner lines) correspond to the resummation uncertainty as defined above. The central values are in good agreement for squark-antisquark and gluino-pair production, and for squark-gluino production at larger masses. For squark-squark production the agreement is less satisfactory, especially for smaller masses. This is consistent with the observation from figure 3 that the NLO corrections for squark-squark production are not as dominated by the threshold contributions as those for the other processes. In all cases, however, the two different NLL predictions are consistent with each other once the uncertainty associated with the resummation procedure is taken into account. It can also be seen that the uncertainty band for the fixed-scale implementation $\mathrm{NLL}_{\text {fixed }}$ is mostly contained inside the uncertainty band of the running-scale result, with the possible exception of the small-mass region. In light of this, in section 3 we will take the matched NLO/NLL result, eq. (2.20), with a running soft scale, eqs. (2.28) and (2.29), as our default and best prediction.

\section{Numerical results}

In this section we present numerical results for the cross sections of the five SUSY processes introduced in section 2.1 and 2.3. In section 3.1 we discuss the impact of the NLL soft and Coulomb corrections on the central value of the total cross sections and the uncertainties for the production of light-flavour squarks and gluinos. In section 3.2 we provide predictions for a selection of the benchmark points defined in [36]. The results for stop-antistop production are presented in 3.3. In order to facilitate the use of our results, the arXiv submission of this paper includes grids with predictions for the LHC with $\sqrt{s}=7$ and $8 \mathrm{TeV}$, for light-flavour squark and gluino masses from $200-2000 \mathrm{GeV}$ and stop masses from $100-1000 \mathrm{GeV}(200-2500 \mathrm{GeV}$ and $100-1200 \mathrm{GeV}$, respectively, for $\sqrt{s}=8 \mathrm{TeV})$. We also provide a Mathematica file containing interpolations of the cross sections with an accuracy 

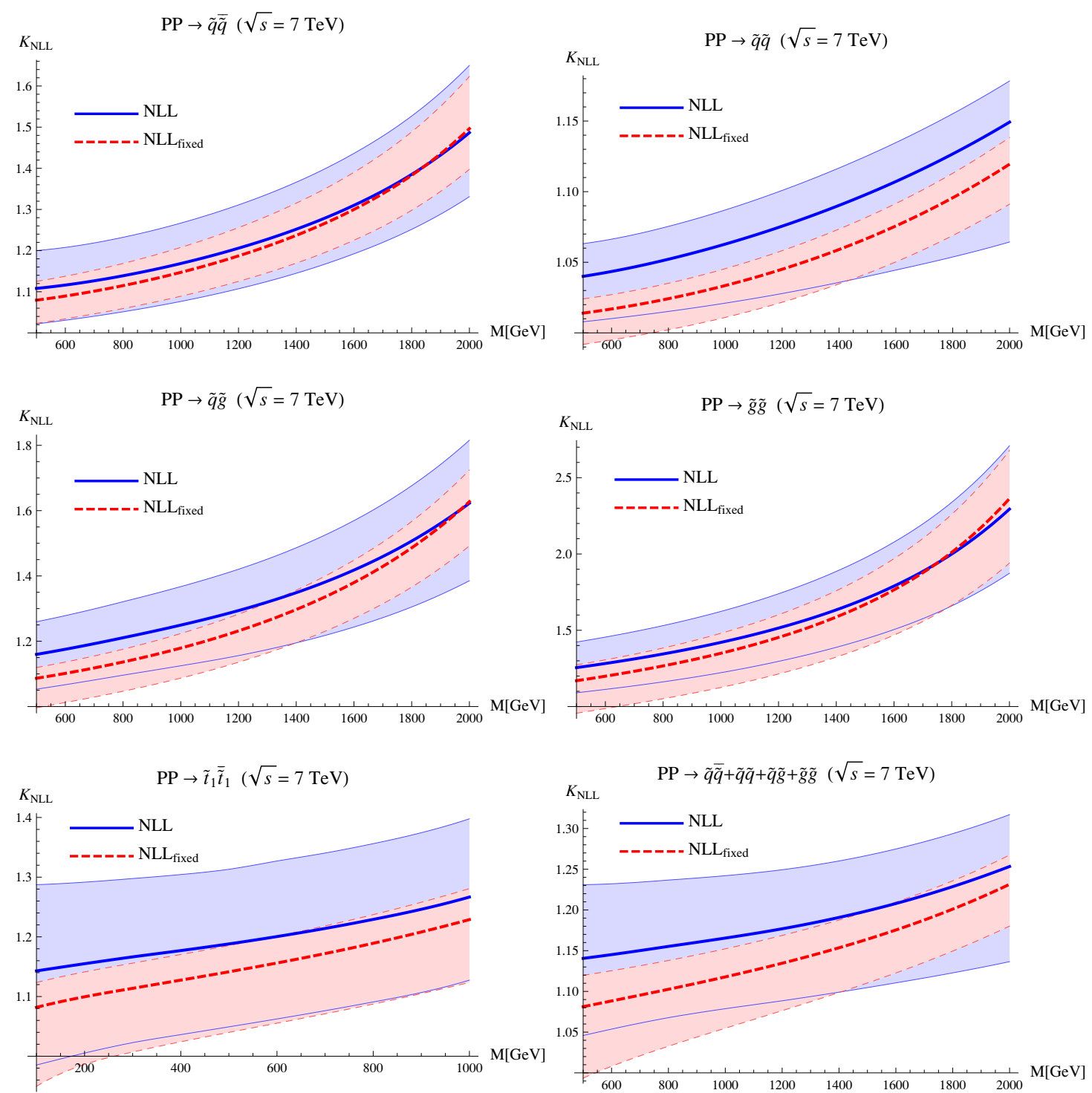

Figure 6. Resummation uncertainty for the NLL resummed result with a running soft scale (NLL, solid blue) and a fixed soft scale ( $\mathrm{NLL}_{\text {fixed }}$, dashed red) for squark-antisquark (top-left), squarksquark (top-right), squark-gluino (centre-left), gluino-gluino (centre-right), stop-antistop (bottomleft) production and the inclusive gluino and light-flavour squark cross section (bottom-right) at LHC with $\sqrt{s}=7 \mathrm{TeV}$. The central line represents the $K$-factor for the default scale choice, while the band gives the resummation uncertainty associated with the result. See text for explanation.

that is typically better than $\sim 1 \%$, and at worst $1-2 \%$ for almost degenerate masses or $2-3 \%$ for the lower boundaries of the intervals where $m_{\tilde{q}}, m_{\tilde{g}}<400 \mathrm{GeV}$.

\subsection{Squark and gluino production at NLL}

To illustrate how different classes of corrections contribute to the total cross section, we introduce three different NLL implementations:

- NLL: our default implementation. Contains the full combined soft and Coulomb 
resummation, eq. (2.18), including bound-state contributions below threshold, eq. (2.17). For the soft scale we adopt the running scale given in eqs. (2.28), (2.29).

- $N L L_{\text {noBS }}$ : as above, but without the inclusion of bound-state effects.

- $N L L_{s+h}$ : this implementation includes resummation of soft and hard logarithms only, without Coulomb resummation. This is obtained using eqs. (2.19) and (2.24).

The three NLL approximations defined above are always matched to the exact NLO results computed with PROSPINO, according to (2.20). As input for the convolution with the parton luminosity functions, eq. (2.3), we adopt the MSTW08NLO PDF set [40] and the associated strong coupling constant $\alpha_{s}\left(M_{Z}\right)=0.1202$. Unless otherwise specified, the parameter $r$, defined as

$$
r=\frac{m_{\tilde{g}}}{m_{\tilde{q}}}
$$

is set to one.

We start presenting results for the NLL $K$-factor, defined as

$$
K_{\mathrm{NLL}}=\frac{\sigma^{\text {matched }}}{\sigma^{\mathrm{NLO}}}
$$

where $\sigma^{\text {matched }}$ is our matched result for one of the NLL implementations defined in the beginning of this section and $\sigma^{\mathrm{NLO}}$ the fixed-order NLO result obtained using PROSPINO. The NLL- $K$-factor for LHC with $7 \mathrm{TeV}$ centre-of-mass energy is plotted in figure 7 , for the four light-squark/gluino production processes and the mass range $m_{\tilde{q}}=m_{\tilde{g}}=500$ $2000 \mathrm{GeV}$. The results for $\sqrt{s}=14 \mathrm{TeV}$ and the mass range $m_{\tilde{q}}=m_{\tilde{g}}=500-3000 \mathrm{GeV}$ are given in figure 8. The NLL corrections for our default implementation (solid blue lines) can be large, with corrections to the fixed-order NLO results of up to $120 \%$ in the upper mass range for gluino-gluino production at $7 \mathrm{TeV}$. The higher-order effects are smaller, but still sizeable, for the other three processes, due to the smaller colour charges involved in squark-antisquark, squark-squark and squark-gluino production. Furthermore, for a fixed SUSY mass the $K_{\mathrm{NLL}}$-factor decreases from 7 to $14 \mathrm{TeV}$, consistently with the expectation that at lower centre-of-mass energies the threshold region plays a more prominent role.

The effect of including Coulomb resummation and its interference with soft resummation is on average as large as (or even larger than) the effect of pure soft and hard corrections, as can be seen comparing our default implementation NLL with $\mathrm{NLL}_{s+h}$ (dashed red lines). Pure soft contributions beyond $\mathcal{O}\left(\alpha_{s}\right)$ amount to $5-60 \%$ of the fixed-order NLO result, depending on the mass and process considered, whereas pure Coulomb effects and interference of soft and Coulomb corrections can amount to up to 60\%. An exception to this is the squark-squark production process, where the effect of Coulomb corrections is small. This particular behaviour originates from cancellations between the cross sections for same-flavour squark production, where the repulsive colour-sextet channel is numerically dominant and gives rise to negative $\mathcal{O}\left(\alpha_{s}^{2} \ln ^{2} \beta / \beta\right)$ corrections, and different-flavour squark production, where the corresponding term is positive, due to the dominance of the attractive colour-triplet channel. 

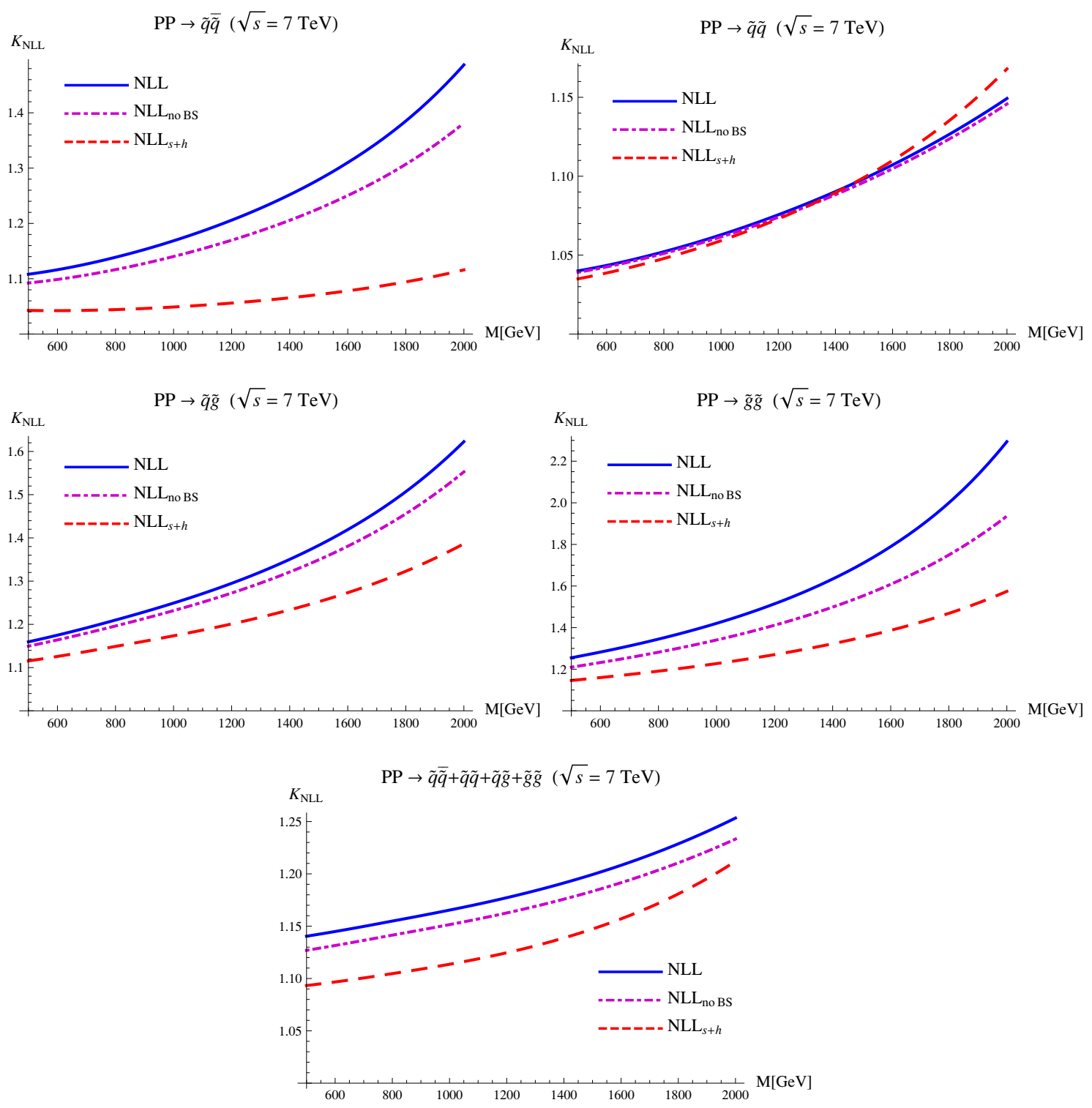

Figure 7. NLL $K$-factor for squark-antisquark (top-left), squark-squark (top-right), squark-gluino (centre-left) and gluino-gluino (centre-right) production at LHC with $\sqrt{s}=7 \mathrm{TeV}$, and for the sum of the four processes (bottom). The plots show $K_{\mathrm{NLL}}$ as a function of $M$ for different NLL approximations: NLL (solid blue), $\mathrm{NLL}_{n o} \mathrm{BS}$ (dot-dashed purple) and $\mathrm{NLL}_{s+h}$ (dashed red). See the text for explanation.

For squark-antisquark, squark-gluino and gluino-gluino production, a significant portion of the total Coulomb and soft-Coulomb corrections originates from bound-state effects below threshold. These correspond to the difference between the NLL and NLL no BS (dotdashed purple) curves in the plots. For squark-antisquark and squark-gluino production bound-state corrections amount to $2-10 \%$ of the fixed-order NLO cross section, whereas for gluino-gluino production they can be as large as $30 \%$. 

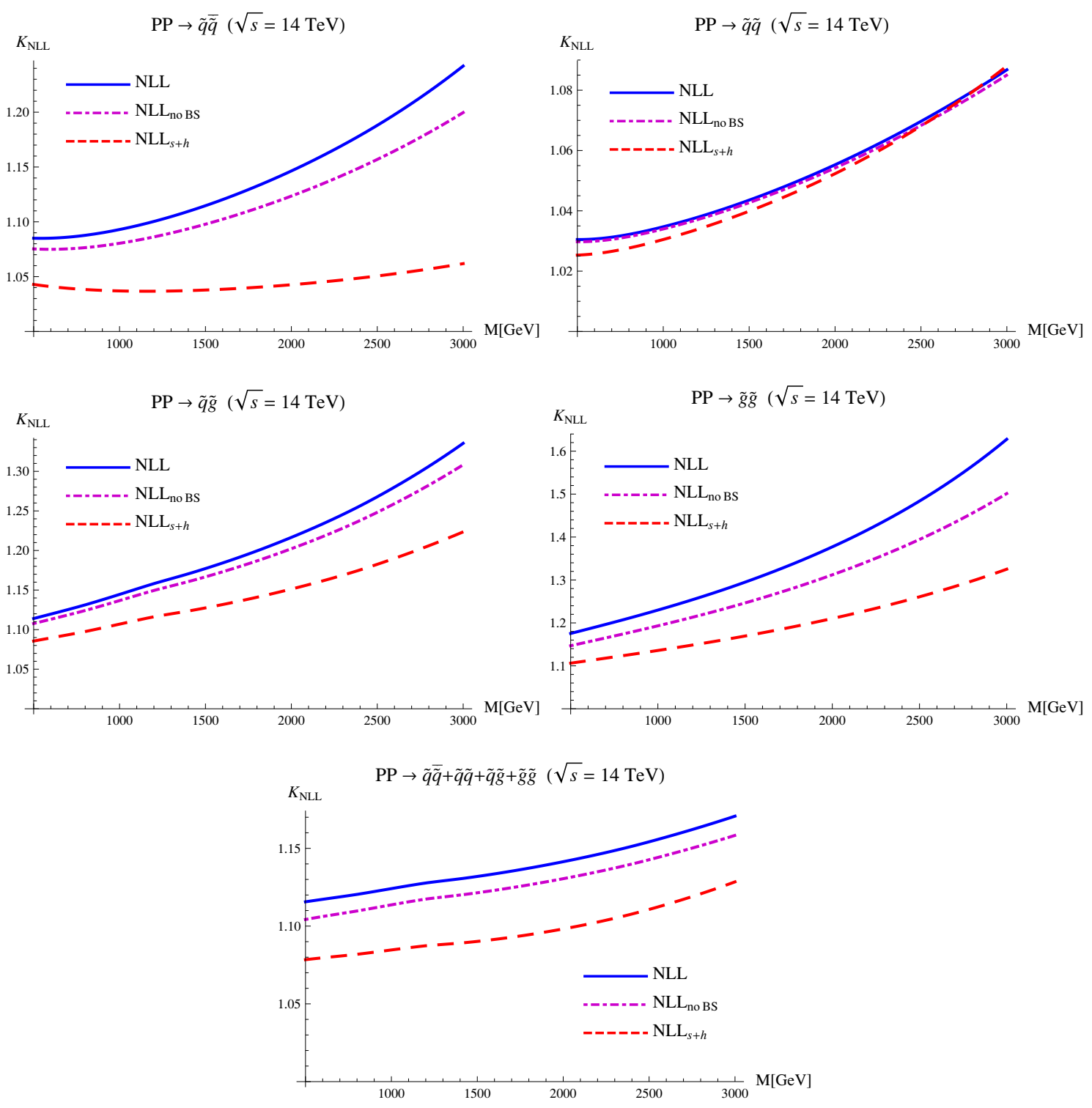

Figure 8. NLL $K$-factor for squark-antisquark (top-left), squark-squark (top-right), squark-gluino (centre-left) and gluino-gluino (centre-right) production at LHC with $\sqrt{s}=14 \mathrm{TeV}$, and for the sum of the four processes (bottom). The plots show $K_{\mathrm{NLL}}$ as a function of $M$ for different NLL approximations: NLL (solid blue), $\mathrm{NLL}_{\text {no BS }}$ (dot-dashed purple) and $\mathrm{NLL}_{s+h}$ (dashed red). See the text for explanation.

Figure 9 shows the NLL $K$-factor for the total SUSY production rate at the $7 \mathrm{TeV}$ LHC as a contour plot in the $\left(m_{\tilde{g}}, m_{\tilde{q}}\right)$-plane. The $r$-dependence of the total resummed cross section arises from an interplay of the $r$-dependence of the single-process cross sections and of the relative dominance of the four subprocesses for a given $r$. The largest $K$ factor is obtained for $m_{\tilde{q}} \sim 2 \mathrm{TeV}$ and $m_{\tilde{g}} \sim 1.4 \mathrm{TeV}$, with corrections of $50 \%$ to the NLO cross section. The plot shows also the recent exclusion limit published by the ATLAS collaboration in [2] assuming a simplified model of a massless neutralino, a gluino octet 


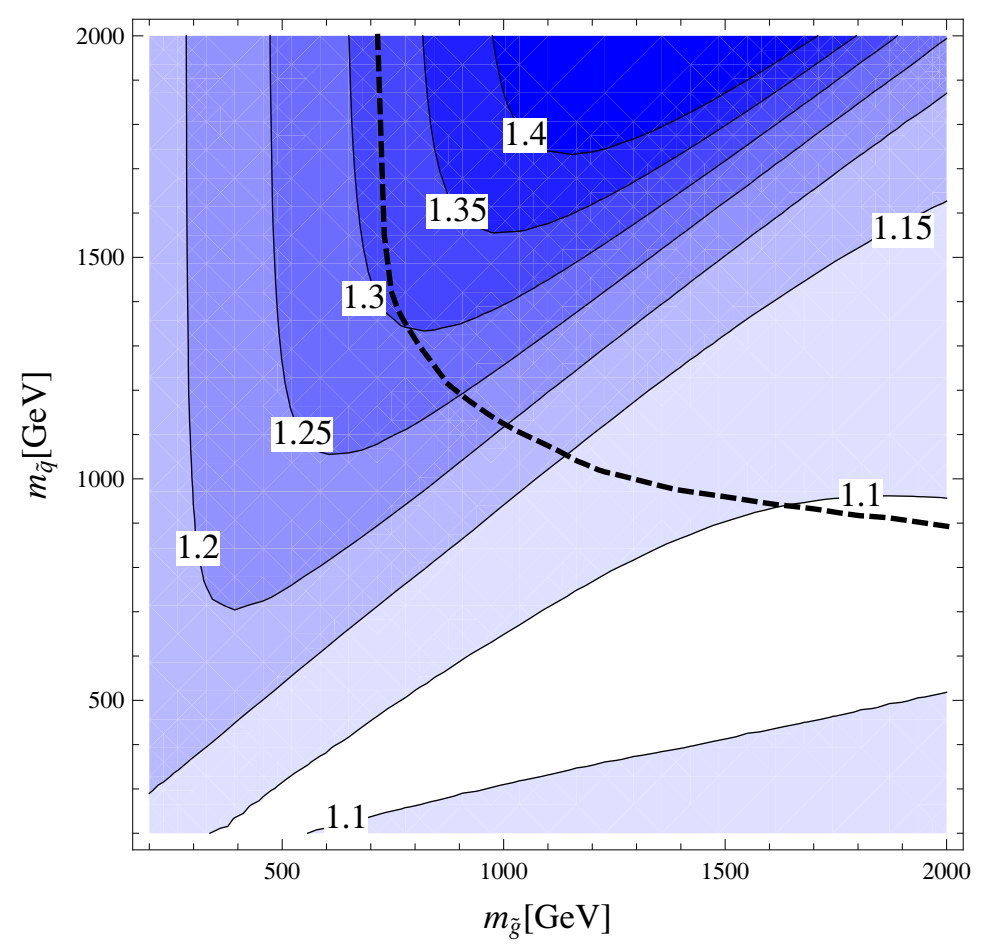

Figure 9. NLL $K$-factor for the total SUSY production rate at LHC with $\sqrt{s}=7 \mathrm{TeV}$ as a function of the gluino mass $m_{\tilde{g}}$ and average squark mass $m_{\tilde{q}}$. The dashed line corresponds to the most recent exclusion limit presented in [2].
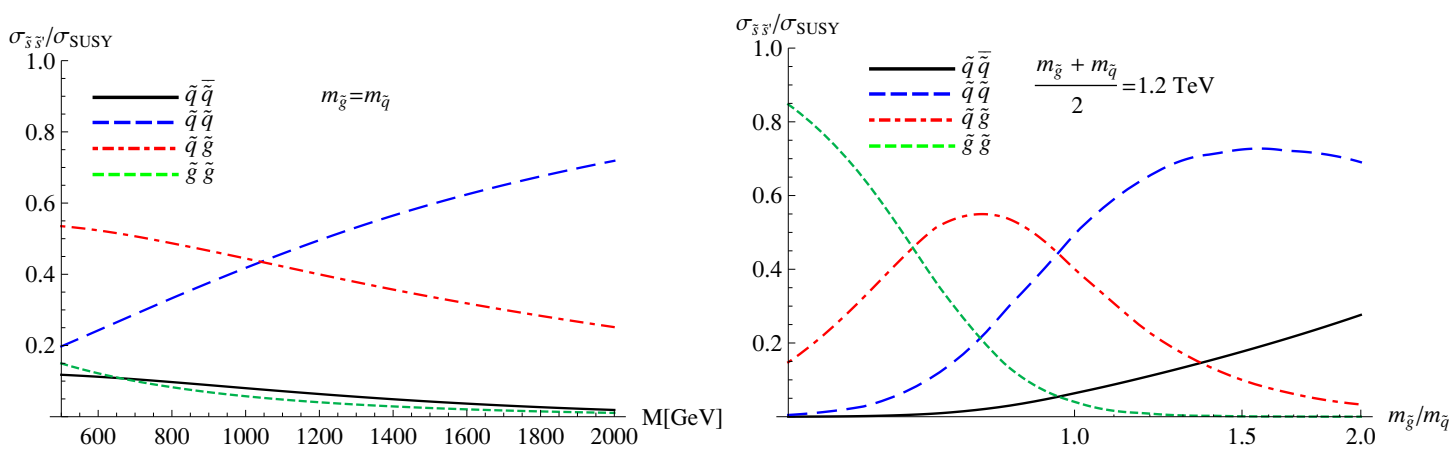

Figure 10. Ratio of the NLL production-cross sections for the processes (2.7) to the total NLL rate of coloured sparticle production $\sigma_{\mathrm{SUSY}}$ for the LHC with $\sqrt{s}=7 \mathrm{TeV}$. Left: Mass dependence for a fixed mass-ratio $m_{\tilde{q}}=m_{\tilde{g}}=M$, Right: Dependence on the ratio $m_{\tilde{g}} / m_{\tilde{q}}$ for a fixed average $\operatorname{mass}\left(m_{\tilde{q}}+m_{\tilde{g}}\right) / 2=1.2 \mathrm{TeV}$.

and degenerate squarks of the first two generations, while all the other supersymmetric particles, including stops and sbottoms, are decoupled by giving them a mass of $5 \mathrm{TeV}$. The limits are therefore not directly comparable to our results which treat the sbottom as degenerate with the light-flavour squarks, but are shown here as an indication of the current LHC reach. We do not attempt to estimate how resummation would affect the determination of this limit. However, one can observe that in the large squark-mass region 
the exclusion limit crosses regions with a $K$-factor bigger than 1.3 , where resummation effects on the limit extraction might be relevant.

Given the large effect of resummation, especially for squark-gluino and gluino-gluino production, it is interesting to study how the relative contribution of the four production processes to the total SUSY production rate is modified by the inclusion of NLL corrections. This is shown in figure 10. The qualitative behaviour of the relative contribution of the four different processes is very similar to the LO result (figure 1). However at large masses one can notice an enhancement of the squark-gluino production rate compared to the squark-squark channel (left plot), as one would expect from the larger NLL $K$-factor for the first processes. For a fixed average squark and gluino mass of $1.2 \mathrm{TeV}$ (right plot) the relative ratios are basically unchanged for moderate values of $r=m_{\tilde{g}} / m_{\tilde{q}}$, though one observes a significant enhancement of the squark-antisquark cross section for large gluino masses $(r=2)$.

As a result of including the threshold-enhanced higher-order corrections, one expects that the uncertainty due to missing perturbative corrections is reduced compared to the NLO results. While for NLO the theoretical uncertainty arises from scale-variation only, the total theoretical error of the NLL results is obtained by adding scale and resummation uncertainties in quadrature, as defined in section 2.4. The uncertainty bands for NLO, $\mathrm{NLL}_{s+h}$ and NLL approximations are shown in figure 11 for the LHC with $\sqrt{s}=7 \mathrm{TeV}$ and in figure 12 for the LHC with $\sqrt{s}=14 \mathrm{TeV}$. In all plots the cross sections are normalized to unity at the central values of the scales and other input parameters. It is evident that the combined resummation of soft and Coulomb effects (NLL, solid blue) generally leads to a significant reduction of theoretical uncertainties compared to the NLO result (dotted black), especially in squark-antisquark and squark-squark production, where the error is reduced by a factor 2 or more in the large-mass region. The behaviour of $\mathrm{NLL}_{s+h}$ (dashed red) is more process-dependent, with basically no uncertainty reduction compared to the fixed-order NLO result for squark-antisquark production, and moderate effects for squarkgluino and gluino-gluino production. For squark-squark production (and, as a consequence of the dominance of squark-squark production, for the total SUSY production rate) the uncertainties of $\mathrm{NLL}_{s+h}$ and NLL are very similar, due to the smallness of Coulomb effects in this particular production channel. The large reduction of the scale dependence for squark-antisquark production by soft-Coulomb interference effects is consistent with recent NNLL studies in this channel [24] that include the first Coulomb correction.

\subsection{Benchmark points for SUSY searches at LHC}

In addition to the grid files provided with the arXiv submission of this paper, we here present numerical predictions for some benchmark points at the LHC with $7 \mathrm{TeV}$ centreof-mass energy, in order to illustrate the effect of our NLL results on the production cross sections. We employ the sets of benchmark points defined in [36], that are compatible with recent LHC bounds and other data such as $b \rightarrow s \gamma$, but not necessarily with constraints from the anomalous magnetic moment of the muon, or from the dark matter relic abundance. We consider the seven lines in the constrained MSSM (CMSSM) parameter space defined in [36] and one line for the minimal gauge mediated SUSY breaking (mGMSB) 

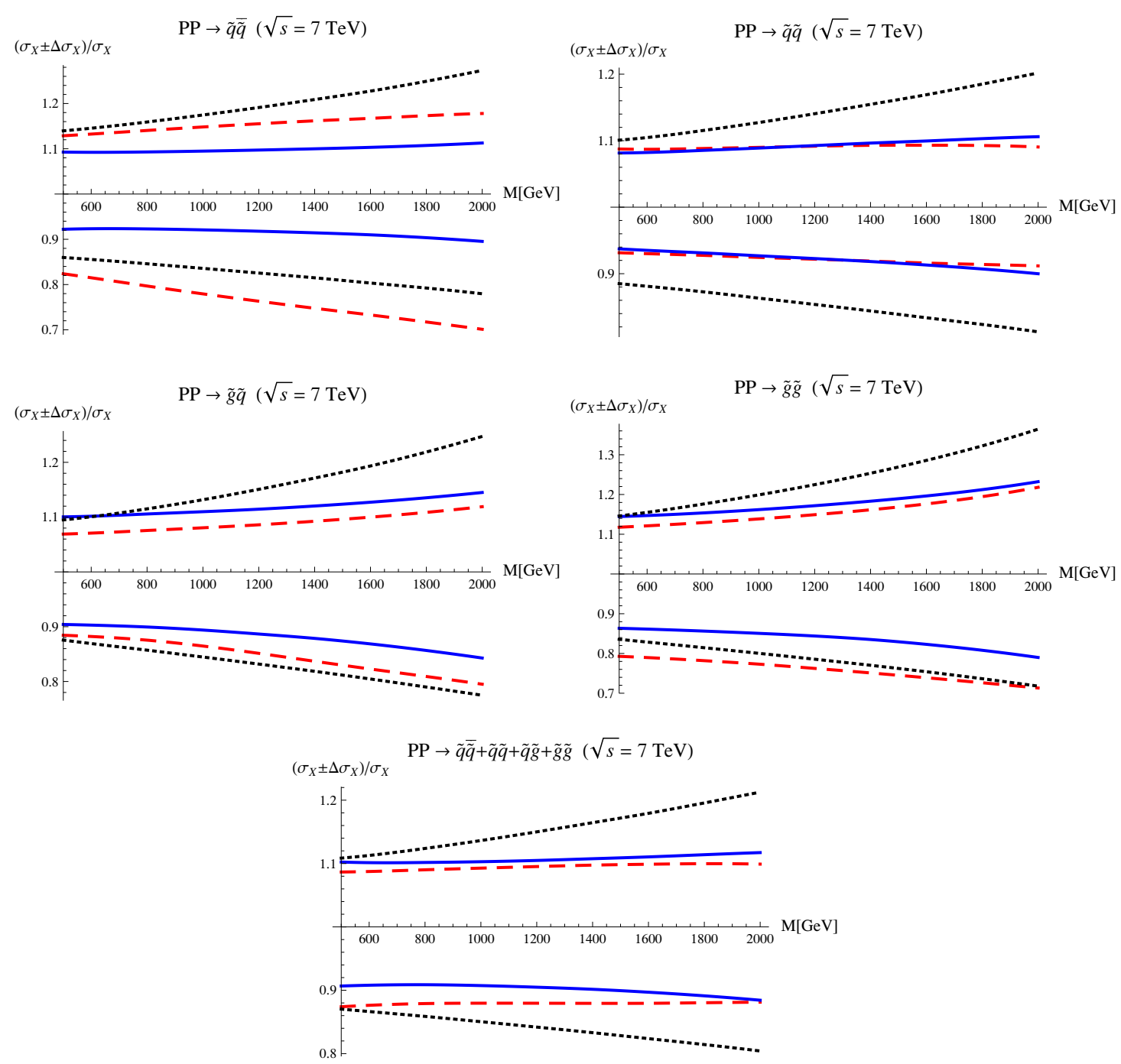

Figure 11. Total theoretical uncertainty of the NLO approximation (dotted black), full NLL resummed result (solid blue) and $\mathrm{NLL}_{s+h}$ (dashed red) at the LHC with $\sqrt{s}=7 \mathrm{TeV}$. All cross sections are normalized to one at the central value of the scales.

scenario. For each line we selected one benchmark point expected to be relevant for $5 \mathrm{fb}^{-1}$ of data and a second point relevant for $10-30 \mathrm{fb}^{-1}$, where we naively extrapolate the reach of the $1 \mathrm{fb}^{-1}$ LHC data $[2,3]$ that exclude CMSSM benchmark points and simplified models with total SUSY production cross sections of the order of $\sigma_{\text {SUSY }} \gtrsim 0.04 \mathrm{pb}$ for $m_{\tilde{q}} \lesssim m_{\tilde{g}}$ and $\sigma_{\text {SUSY }} \gtrsim 0.1 \mathrm{pb}$ for $m_{\tilde{q}}>m_{\tilde{g}}$. The mGMSB scenario we have selected has a quasi-stable neutralino as next-to-lightest SUSY particle (NLSP), so a similar reach as for CMSSM-type scenarios are expected. Since only the squark and gluino masses are relevant for the production cross sections, we have chosen points with a reasonable spread of masses and mass ratios, covering the range of average sparticle masses, $1.3 \mathrm{TeV} \lesssim\left(m_{\tilde{g}}+m_{\tilde{q}}\right) / 2 \lesssim 1.5 \mathrm{TeV}$, and the mass ratios $0.75 \lesssim \frac{m_{\tilde{g}}}{m_{\tilde{q}}} \lesssim 1.12$. This mass range is also compatible with the estimated discovery reach [52] of $m_{\tilde{g}} \sim 1.3 \mathrm{TeV}(1.6 \mathrm{TeV})$ for $m_{\tilde{q}} \sim m_{\tilde{g}}$ at $5 \mathrm{fb}^{-1}\left(30 \mathrm{fb}^{-1}\right)$ and 


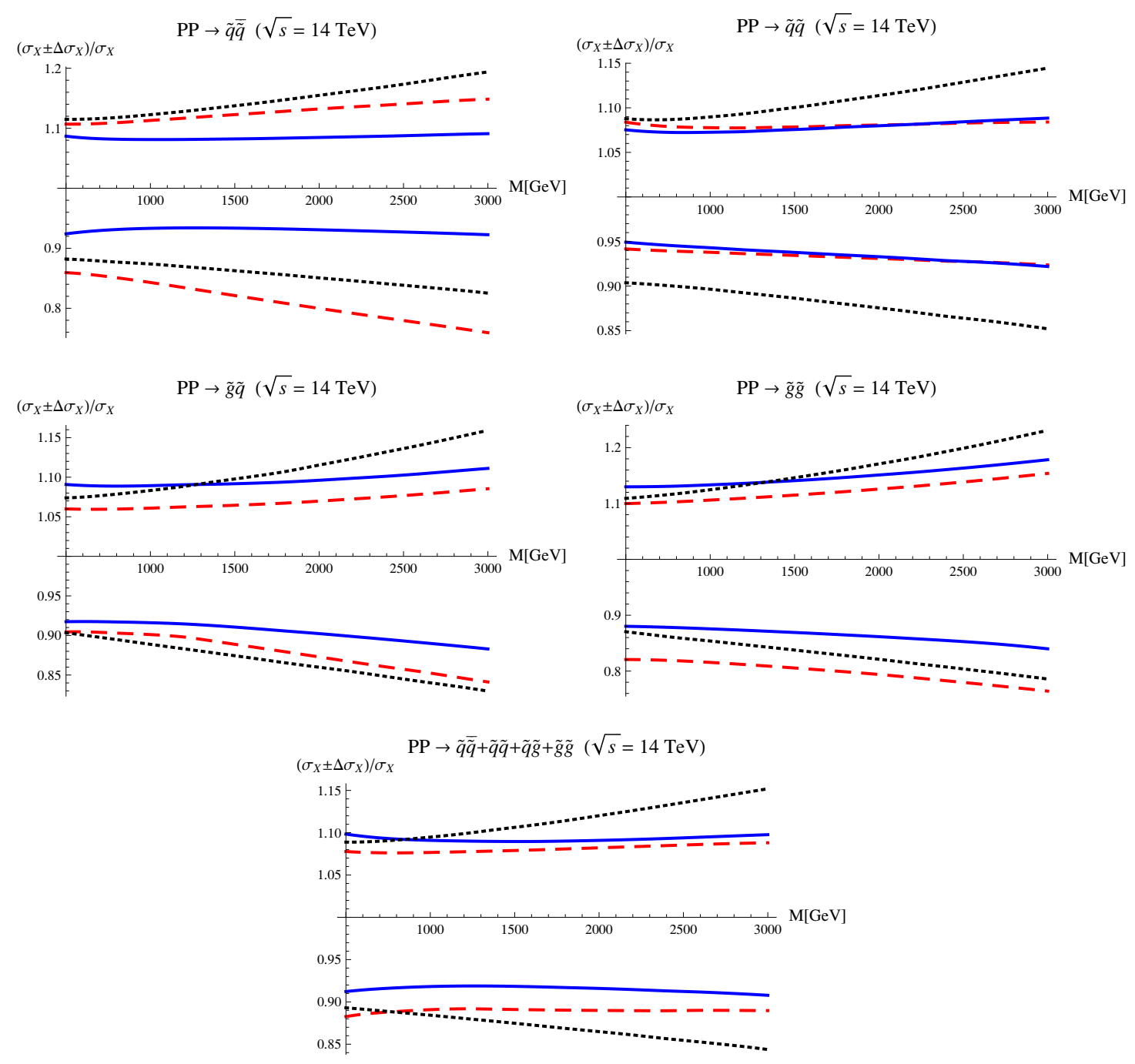

Figure 12. Total theoretical uncertainty of the NLO approximation (dotted black), full NLL resummed result (solid blue) and $\mathrm{NLL}_{s+h}$ (dashed red) at the LHC with $\sqrt{s}=14 \mathrm{TeV}$. All cross sections are normalized to one at the central value of the scales.

$m_{\tilde{g}} \sim 0.8 \mathrm{TeV}(1 \mathrm{TeV})$ for $m_{\tilde{q}} \gg m_{\tilde{g}}$ in a CMSSM scenario with $\tan \beta=0.45, A_{0}=0$. The remaining families of benchmark scenarios introduced in [36] tend to have very similar mass ratios as our selected points. Therefore the relative contributions of the different production channels and the effect of the higher-order QCD corrections will be similar, although the decay chains and the resulting collider signatures can be very different. For some scenarios lighter squarks and gluinos than the ones considered here might still be allowed, for instance in GMSB with a stau NLSP. Predictions for such scenarios can be obtained by an interpolation of the grid files provided with the arXiv submission of this paper.

The SUSY breaking parameters and the resulting mass spectrum of the coloured SUSY particles for the selected points is shown in tables 2, 3 and 4 together with our best NLL predictions for the total cross section for light-flavour squark and gluino production (including simultaneous soft-gluon and Coulomb resummation as well as bound-state effects). 


\begin{tabular}{|c|c|c|c|c|c|c|c|c|}
\hline & & & & \multicolumn{5}{|c|}{$\sigma_{\mathrm{SUSY}}(\mathrm{pb}), \sqrt{s}=7 \mathrm{TeV}$} \\
Point & $m_{0}$ & $m_{1 / 2}$ & $m_{\tilde{g}}$ & $m_{\tilde{q}}$ & $\mathrm{NLO}$ & $\mathrm{NLL}$ & $\Delta_{\mathrm{PDF}}$ & $K_{\mathrm{NLL}}$ \\
\hline 10.1 .3 & 150 & 600 & 1357 & 1209 & $0.91_{-0.14}^{+0.13} \times 10^{-2}$ & $1.04_{-0.09}^{+0.10} \times 10^{-2}$ & ${ }_{-2.9 \%}^{+4.2 \%}$ & 1.15 \\
\hline 10.1 .4 & 162.5 & 650 & 1461 & 1300 & $4.17_{-0.64}^{+0.60} \times 10^{-3}$ & $4.79_{-0.42}^{+0.45} \times 10^{-3}$ & ${ }_{-3.0 \%}^{+4.2 \%}$ & 1.15 \\
\hline 10.2 .2 & 225 & 550 & 1255 & 1130 & $1.83_{-0.27}^{+0.25} \times 10^{-2}$ & $2.10_{-0.18}^{+0.20} \times 10^{-2}$ & ${ }_{-3.0 \%}^{+4.2 \%}$ & 1.15 \\
\hline 10.2 .5 & 300 & 700 & 1569 & 1412 & $1.66_{-0.27}^{+0.26} \times 10^{-3}$ & $1.92_{-0.17}^{+0.18} \times 10^{-3}$ & ${ }_{-3.1 \%}^{+4.4 \%}$ & 1.16 \\
\hline 10.3 .2 & 350 & 525 & 1209 & 1115 & $2.21_{-0.33}^{+0.30} \times 10^{-2}$ & $2.54_{-0.23}^{+0.24} \times 10^{-2}$ & ${ }_{-3.2 \%}^{+4.4 \%}$ & 1.15 \\
\hline 10.3 .3 & 400 & 600 & 1367 & 1261 & $6.32_{-0.98}^{+0.92} \times 10^{-3}$ & $7.31_{-0.66}^{+0.71} \times 10^{-3}$ & ${ }_{-3.3 \%}^{+4.4 \%}$ & 1.16 \\
\hline 10.4 .2 & 850 & 400 & 983 & 1160 & $3.48_{-0.59}^{+0.57} \times 10^{-2}$ & $4.24_{-0.43}^{+0.48} \times 10^{-2}$ & ${ }_{-6.2 \%}^{+7.0 \%}$ & 1.22 \\
\hline 10.4 .4 & 1050 & 500 & 1207 & 1427 & $3.65_{-0.66}^{+0.68} \times 10^{-3}$ & $4.59_{-0.50}^{+0.54} \times 10^{-3}$ & ${ }_{-7.4 \%}^{+8.5 \%}$ & 1.26 \\
\hline
\end{tabular}

Table 2. CMSSM benchmark points and total inclusive SUSY production cross sections for $\tan \beta=$ $10, A_{0}=0$. All masses are given in $\mathrm{GeV}$.

\begin{tabular}{|c|c|c|c|c|c|c|c|c|}
\hline \multirow[b]{2}{*}{ Point } & \multirow[b]{2}{*}{$m_{0}$} & \multirow[b]{2}{*}{$m_{1 / 2}$} & \multirow[b]{2}{*}{$m_{\tilde{g}}$} & \multirow[b]{2}{*}{$m_{\tilde{q}}$} & \multicolumn{3}{|c|}{$\sigma_{\mathrm{SUSY}}(\mathrm{pb}), \sqrt{s}=7 \mathrm{TeV}$} & \multirow[b]{2}{*}{$K_{\mathrm{NLL}}$} \\
\hline & & & & & NLO & NLL & $\Delta_{\mathrm{PDF}}$ & \\
\hline 40.1 .2 & 345 & 550 & 1259 & 1144 & $1.66_{-0.25}^{+0.23} \times 10^{-2}$ & $1.91_{-0.17}^{+0.18} \times 10^{-2}$ & $\begin{array}{l}+4.3 \% \\
-3.1 \% \\
\end{array}$ & 1.15 \\
\hline 40.1 .4 & 375 & 650 & 1468 & 1325 & $3.48_{-0.55}^{+0.52} \times 10^{-3}$ & $4.02_{-0.36}^{+0.38} \times 10^{-3}$ & $\begin{array}{l}+4.4 \% \\
-3.1 \% \\
\end{array}$ & 1.15 \\
\hline 40.2 .2 & 600 & 500 & 1172 & 1153 & $1.90_{-0.29}^{+0.28} \times 10^{-2}$ & $2.22_{-0.21}^{+0.23} \times 10^{-2}$ & $\begin{array}{l}+5.0 \% \\
-3.8 \%\end{array}$ & 1.17 \\
\hline 40.2 .5 & 750 & 650 & 1492 & 1460 & $1.34_{-0.23}^{+0.22} \times 10^{-3}$ & $1.59_{-0.16}^{+0.17} \times 10^{-3}$ & $\begin{array}{l}+5.3 \% \\
-4.0 \% \\
\end{array}$ & 1.19 \\
\hline 40.3 .1 & 1000 & 350 & 886 & 1182 & $5.30_{-0.94}^{+0.96} \times 10^{-2}$ & $6.63_{-0.71}^{+0.77} \times 10^{-2}$ & $\begin{array}{l}+8.4 \% \\
-7.8 \% \\
\end{array}$ & 1.25 \\
\hline 40.3 .5 & 1200 & 450 & 1111 & 1446 & $5.29_{-1.00}^{+1.06} \times 10^{-3}$ & $6.86_{-0.77}^{+0.83} \times 10^{-3}$ & $\begin{array}{l}+10 \% \\
-9.3 \% \\
\end{array}$ & 1.30 \\
\hline
\end{tabular}

Table 3. CMSSM benchmark points and total inclusive SUSY production cross sections for $\tan \beta=$ $40, A_{0}=-500 \mathrm{GeV}$. All masses are given in $\mathrm{GeV}$.

Here $m_{\tilde{q}}$ denotes the average mass of all squarks except the stops, following the setup of $[24,53]$. The low-scale mass parameters have been generated using SUSY-HIT [54] employing SuSpect2.41 [55] with the standard model input $m_{t}=172.5 \mathrm{GeV}, \alpha_{s}\left(m_{Z}\right)=0.1172$, $\bar{m}_{b}\left(m_{b}\right)=4.25 \mathrm{GeV} .^{7}$ The cross sections for the separate squark and gluino production processes are shown in tables 5 and 6 . The stops are always heavier than $m_{\tilde{t}_{1}}>750 \mathrm{GeV}$ for the considered benchmark points so direct stop-antistop production will be out of reach at the LHC with $\sqrt{s}=7 \mathrm{TeV}$ (for some of the benchmark points, it might be possible to discover them in the gluino-decay products). Therefore we will give results for stop-antistop production separately in section 3.3.

\footnotetext{
${ }^{7}$ Note that in the mass-spectra quoted in [36] the masses are rounded to $5 \mathrm{GeV}$ accuracy and only the light-flavour squark masses are averaged. Furthermore version 2.3 of SuSpect has been used for the CMSSM benchmark points and SOFTSUSY for the mGMSB benchmark points.
} 


\begin{tabular}{|c|c|c|c|c|c|c|c|}
\hline & & & & \multicolumn{2}{|c|}{$\sigma_{\text {SUSY }}(\mathrm{pb}), \sqrt{s}=7 \mathrm{TeV}$} \\
Point & $\Lambda_{\text {SUSY }}$ & $m_{\tilde{g}}$ & $m_{\tilde{q}}$ & NLO & NLL & $\Delta_{\text {PDF }}$ & $K_{\text {NLL }}$ \\
\hline mGMSB2.2.1 & $1.2 \times 10^{5}$ & 943 & 1142 & $4.55_{-0.77}^{+0.76} \times 10^{-2}$ & $5.57_{-0.57}^{+0.63} \times 10^{-2}$ & ${ }_{-6.4 \%}^{+7.3 \%}$ & 1.22 \\
\hline mGMSB2.2.4 & $1.5 \times 10^{5}$ & 1154 & 1408 & $5.04_{-0.92}^{+0.95} \times 10^{-3}$ & $6.38_{-0.69}^{+0.76} \times 10^{-3}$ & ${ }_{-7.9 \%}^{+8.9 \%}$ & 1.27 \\
\hline
\end{tabular}

Table 4. mGMSB benchmark points and total inclusive SUSY production cross sections for $\tan \beta=$ $15, N_{\text {mess }}=1, M_{\text {mess }}=10^{9} \mathrm{GeV}$. All masses and scales in GeV.

For comparison, NLO results obtained using PROSPINO are also shown in the tables. Our setup agrees with the one in $[18,53]$ and the NLO results agree at the expected one-percent level or better with the results obtained from an interpolated grid using NLL-fast [53]. For the NLO results the scale uncertainty is estimated by varying the factorisation scale in the interval $M / 2<\mu_{f}<M$, with the renormalization scale set equal to the factorization scale. The tables also include the relative PDF uncertainties of the NLO cross sections. Based on experience with top-pair production [32], we expect that these agree with the relative PDF uncertainties of the NLL results at the relevant accuracy. Note that, due to correlations, the PDF uncertainty of the total SUSY production cross section is not equal to the sum of the uncertainties of the individual channels [18]. For the NLL results we quote the total uncertainty including scale and resummation uncertainties, as discussed in section 2.4. In this case, the uncertainty of the total SUSY production cross section was obtained by neglecting correlations and adding the uncertainties of the individual production channels linearly, which gives a conservative upper bound.

As can be seen from tables 2, 3 and 4 , for the benchmark points with $m_{\tilde{q}} \lesssim m_{\tilde{g}}$ the NLL correction to the inclusive squark and gluino production cross section is in the 15$19 \%$-range, which is consistent with figure 9 . For these points the theoretical uncertainty is reduced from $\pm 14-15 \%$ at NLO to $\pm 9-10 \%$ at NLL, in agreement with the behaviour seen in figure 11. The PDF uncertainty is also small for the benchmark points with $m_{\tilde{q}}<m_{\tilde{g}}$, since only the relatively precisely known quark PDFs are relevant at leading order for the dominant squark-squark production channel. For scenarios where the gluinos are lighter than the squarks, the NLL corrections grow to $20-30 \%$ and the theoretical uncertainty at NLL is at the 11-12\% level. These features can be understood by considering the relative contributions of the different production processes in figure 10 and tables 5 and 6 . For $m_{\tilde{q}} \lesssim m_{\tilde{g}}$, squark-pair production with moderate NLL corrections in the $6-10 \%$ range is the dominant production channel, with a non-negligible contribution from squark-gluino production with larger NLL corrections of the order of $30 \%$. For $m_{\tilde{g}}<m_{\tilde{q}}$ the roles are reversed, resulting in larger NLL corrections to the total rate and a slightly larger theoretical uncertainty, as can be seen from the uncertainties of the different production channels in figure 11. Due to the large uncertainties in the current gluon PDF sets for large $x$, the PDF error for these points rises to the 10\%-level. In gluino-pair production, especially for large gluino masses, the PDF error can even become $\sim 30 \%$. At a mass ratio $\frac{m_{\tilde{g}}}{m_{\tilde{q}}} \lesssim 0.75$, as realized for the 40.3 .1 and 40.3 .5 points, gluino-pair production overtakes squark-pair production as the second-most important channel. For these points, the gluinos 

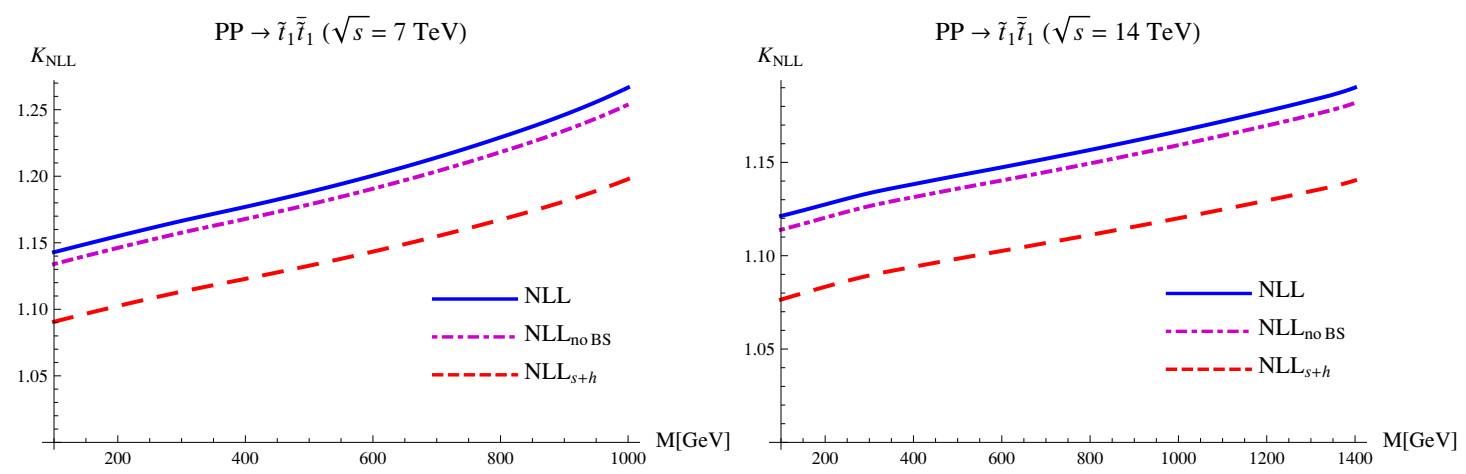

Figure 13. $K_{\mathrm{NLL}}$ for stop-antistop production at the LHC at $\sqrt{s}=7 \mathrm{TeV}$ (left) and $\sqrt{s}=14 \mathrm{TeV}$

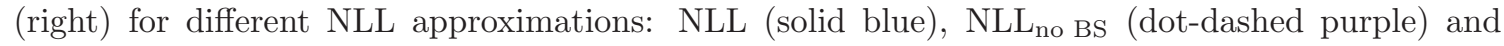
$\mathrm{NLL}_{s+h}$ (dashed red).
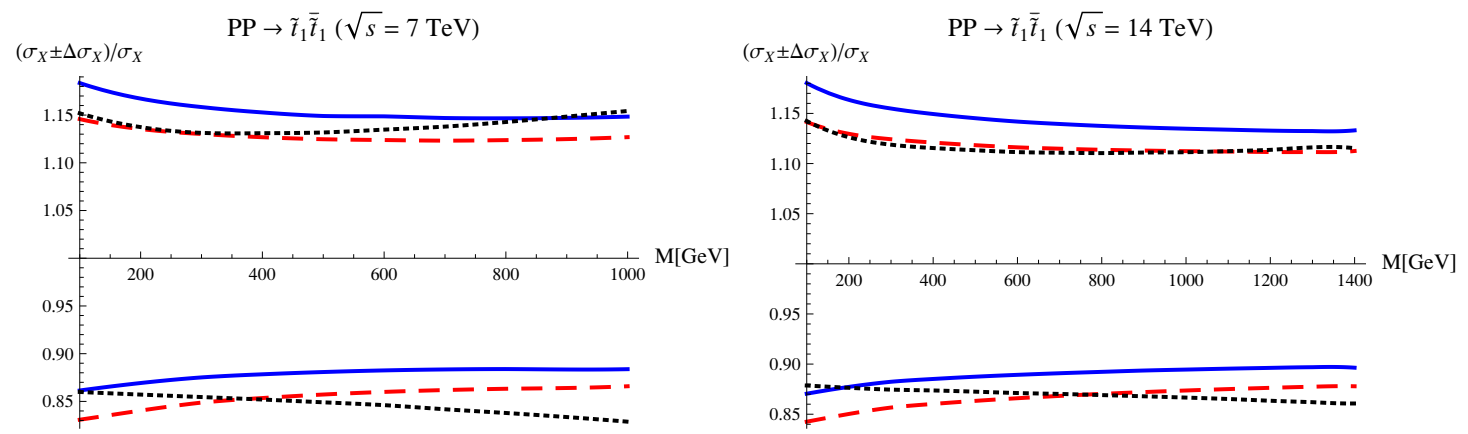

Figure 14. Total theoretical uncertainty of the NLO approximation (dotted black), full NLL resummed result (solid blue) and $\mathrm{NLL}_{s+h}$ (dashed red) for $\tilde{t}_{1} \overline{\tilde{t}}_{1}$ at the LHC with $\sqrt{s}=7 \mathrm{TeV}$ (left) and $\sqrt{s}=14 \mathrm{TeV}$ (right). All cross sections are normalized to one at the central value of the scales.

are relatively light, and the NLL corrections to gluino-pair production are at most $45 \%$. For benchmark points with heavier gluinos, the NLL corrections can grow to 70-80\%, but the gluino-pair production rate is negligible for these points. For the moderate mass ratios considered here, squark-antisquark production is always much suppressed, but would have the second-largest cross section for $\frac{m_{\tilde{g}}}{m_{\tilde{q}}} \rightarrow 2$.

\subsection{Results for stop-antistop production}

In this section we present results for resummation for stop-antistop production $P P \rightarrow \tilde{t}_{i} \overline{\tilde{t}}_{i}$. At LO the cross section depends only on the stop mass, while at NLO it also presents a (much smaller) dependence on the mixing angle $\theta_{\tilde{t}}$ and the squark and gluino masses. For definiteness, we follow [18] and fix all the parameters, except for the stop mass, to the values corresponding to the CMSSM benchmark point 40.2.4 with SUSY breaking parameters $m_{0}=700 \mathrm{GeV}, m_{1 / 2}=600 \mathrm{GeV}, A_{0}=-500 \mathrm{GeV}$ and $\tan \beta=40$ [36]. Using SUSY-HIT [54] we obtain the relevant input parameters $m_{\tilde{g}}=1386 \mathrm{GeV}, m_{\tilde{q}}=1358$ and $\cos \theta_{\tilde{t}}=0.39$. The numerical dependence on these parameters has been studied in [17] and 


\begin{tabular}{|c|c|c|c|c|c|}
\hline & & \multicolumn{4}{|c|}{$\sigma\left(p p \rightarrow \tilde{s} \tilde{s}^{\prime}\right)(\mathrm{pb}), \sqrt{s}=7 \mathrm{TeV}$} \\
\hline Point & $\tilde{s} \tilde{s}^{\prime}$ & NLO & NLL & $\Delta_{\mathrm{PDF}}$ & $K_{\mathrm{NLL}}$ \\
\hline \multirow[t]{4}{*}{10.1 .3} & $\tilde{q} \overline{\tilde{q}}$ & $7.26_{-1.25}^{+1.34} \times 10^{-4}$ & $8.80_{-0.73}^{+0.84} \times 10^{-4}$ & & 1.21 \\
\hline & $\tilde{q} \tilde{q}$ & $6.22_{-0.88}^{+0.81} \times 10^{-3}$ & $6.72_{-0.48}^{+0.55} \times 10^{-3}$ & & 1.08 \\
\hline & $\tilde{q} \tilde{g}$ & $2.06_{-0.35}^{+0.30} \times 10^{-3}$ & $2.69_{-0.32}^{+0.32} \times 10^{-3}$ & & 1.31 \\
\hline & $\tilde{g} \tilde{g}$ & $0.77_{-0.17}^{+0.17} \times 10^{-4}$ & $1.26_{-0.22}^{+0.24} \times 10^{-4}$ & & 1.64 \\
\hline \multirow[t]{4}{*}{ 10.1.4 } & $\tilde{q} \overline{\tilde{q}}$ & $2.91_{-0.52}^{+0.56} \times 10^{-4}$ & $3.59_{-0.31}^{+0.35} \times 10^{-4}$ & & 1.23 \\
\hline & $\tilde{q} \tilde{q}$ & $3.02_{-0.44}^{+0.41} \times 10^{-3}$ & $3.28_{-0.24}^{+0.27} \times 10^{-3}$ & & 1.09 \\
\hline & $\tilde{q} \tilde{g}$ & $0.83_{-0.15}^{+0.13} \times 10^{-3}$ & $1.11_{-0.14}^{+0.13} \times 10^{-3}$ & & 1.33 \\
\hline & $\tilde{g} \tilde{g}$ & $2.54_{-0.57}^{+0.59} \times 10^{-5}$ & $4.36_{-0.81}^{+0.87} \times 10^{-5}$ & & 1.72 \\
\hline \multirow[t]{4}{*}{10.2 .2} & $\tilde{q} \overline{\tilde{q}}$ & $1.60_{-0.27}^{+0.29} \times 10^{-3}$ & $1.92_{-0.16}^{+0.18} \times 10^{-3}$ & & 1.20 \\
\hline & $\tilde{q} \tilde{q}$ & $1.17_{-0.16}^{+0.15} \times 10^{-2}$ & $1.25_{-0.09}^{+0.10} \times 10^{-2}$ & & 1.07 \\
\hline & $\tilde{q} \tilde{g}$ & $4.81_{-0.79}^{+0.67} \times 10^{-3}$ & $6.18_{-0.72}^{+0.71} \times 10^{-3}$ & & 1.28 \\
\hline & $\tilde{g} \tilde{g}$ & $2.27_{-0.48}^{+0.48} \times 10^{-4}$ & $3.56_{-0.61}^{+0.65} \times 10^{-4}$ & & 1.57 \\
\hline \multirow[t]{4}{*}{10.2 .5} & $\tilde{q} \overline{\tilde{q}}$ & $0.96_{-0.18}^{+0.19} \times 10^{-4}$ & $1.21_{-0.10}^{+0.12} \times 10^{-4}$ & & 1.26 \\
\hline & $\tilde{q} \tilde{q}$ & $1.26_{-0.19}^{+0.18} \times 10^{-3}$ & $1.38_{-0.11}^{+0.12} \times 10^{-3}$ & & 1.10 \\
\hline & $\tilde{q} \tilde{g}$ & $3.00_{-0.55}^{+0.51} \times 10^{-4}$ & $4.11_{-0.53}^{+0.51} \times 10^{-4}$ & & 1.37 \\
\hline & $\tilde{g} \tilde{g}$ & $0.80_{-0.19}^{+0.20} \times 10^{-5}$ & $1.44_{-0.28}^{+0.30} \times 10^{-5}$ & & 1.81 \\
\hline \multirow[t]{4}{*}{10.3 .2} & $\tilde{q} \overline{\tilde{q}}$ & $1.88_{-0.32}^{+0.34} \times 10^{-3}$ & $2.24_{-0.18}^{+0.21} \times 10^{-3}$ & & 1.19 \\
\hline & $\tilde{q} \tilde{q}$ & $1.33_{-0.19}^{+0.17} \times 10^{-2}$ & $1.43_{-0.10}^{+0.12} \times 10^{-2}$ & & 1.07 \\
\hline & $\tilde{q} \tilde{g}$ & $6.49_{-1.05}^{+0.89} \times 10^{-3}$ & $8.30_{-0.95}^{+0.94} \times 10^{-3}$ & & 1.28 \\
\hline & $\tilde{g} \tilde{g}$ & $3.70_{-0.77}^{+0.77} \times 10^{-4}$ & $5.69_{-0.96}^{+1.03} \times 10^{-4}$ & & 1.54 \\
\hline \multirow[t]{4}{*}{10.3 .3} & $\tilde{q} \overline{\tilde{q}}$ & $4.40_{-0.78}^{+0.85} \times 10^{-4}$ & $5.37_{-0.45}^{+0.52} \times 10^{-4}$ & & 1.22 \\
\hline & $\tilde{q} \tilde{q}$ & $4.23_{-0.62}^{+0.58} \times 10^{-3}$ & $4.58_{-0.34}^{+0.39} \times 10^{-3}$ & $\begin{array}{l}+3 . \\
-2 .\end{array}$ & 1.08 \\
\hline & $\tilde{q} \tilde{g}$ & $1.58_{-0.27}^{+0.24} \times 10^{-3}$ & $2.08_{-0.25}^{+0.24} \times 10^{-3}$ & & 1.32 \\
\hline & $\tilde{g} \tilde{g}$ & $0.69_{-0.15}^{+0.16} \times 10^{-4}$ & $1.13_{-0.21}^{+0.22} \times 10^{-4}$ & & 1.64 \\
\hline \multirow[t]{4}{*}{10.4 .2} & $\tilde{q} \overline{\tilde{q}}$ & $1.25_{-0.21}^{+0.22} \times 10^{-3}$ & $1.48_{-0.12}^{+0.14} \times 10^{-3}$ & & 1.19 \\
\hline & $\tilde{q} \tilde{q}$ & $1.09_{-0.16}^{+0.16} \times 10^{-2}$ & $1.16_{-0.10}^{+0.12} \times 10^{-2}$ & & 1.07 \\
\hline & $\tilde{q} \tilde{g}$ & $1.82_{-0.31}^{+0.29} \times 10^{-2}$ & $2.30_{-0.23}^{+0.24} \times 10^{-2}$ & & 1.27 \\
\hline & $\tilde{g} \tilde{g}$ & $4.55_{-0.94}^{+0.99} \times 10^{-3}$ & $6.37_{-0.88}^{+0.95} \times 10^{-3}$ & $\begin{array}{l}-8.0 \% \\
+17 \% \\
-17 \%\end{array}$ & 1.40 \\
\hline \multirow[t]{4}{*}{ 10.4.4 } & $\tilde{q} \overline{\tilde{q}}$ & $0.85_{-0.16}^{+0.17} \times 10^{-4}$ & $1.07_{-0.09}^{+0.10} \times 10^{-4}$ & $\begin{array}{l}+15 \\
-15\end{array}$ & 1.25 \\
\hline & $\tilde{q} \tilde{q}$ & $1.29_{-0.21}^{+0.22} \times 10^{-3}$ & $1.40_{-0.13}^{+0.16} \times 10^{-3}$ & & 1.08 \\
\hline & $\tilde{q} \tilde{g}$ & $1.86_{-0.34}^{+0.35} \times 10^{-3}$ & $2.47_{-0.27}^{+0.28} \times 10^{-3}$ & & 1.33 \\
\hline & $\tilde{g} \tilde{g}$ & $4.12_{-0.91}^{+1.02} \times 10^{-4}$ & $6.19_{-0.90}^{+0.99} \times 10^{-4}$ & $\begin{array}{l}+22 \% \\
-20 \%\end{array}$ & 1.50 \\
\hline
\end{tabular}

Table 5. NLL results for CMSSM benchmark points for $\tan \beta=10, A_{0}=0$.

found to be negligible. As discussed in section 2.3 we will limit ourselves to results for the lighter mass-eigenstate $\tilde{t}_{1}$.

Numerical results for the lightest stop mass eigenstates for masses in the $100-400 \mathrm{GeV}$ range are presented in table 7 . The NLL $K$-factor is plotted as a function of the mass in figure 13. For the stop-mass range considered here the resummation effects are in the 15- 


\begin{tabular}{|c|c|c|c|c|c|}
\hline & & \multicolumn{4}{|c|}{$\sigma\left(p p \rightarrow \tilde{s} \tilde{s}^{\prime}\right)(\mathrm{pb}), \sqrt{s}=7 \mathrm{TeV}$} \\
\hline Point & $\tilde{s} \tilde{s}^{\prime}$ & NLO & NLL & $\Delta_{\mathrm{PDF}}$ & $K_{\mathrm{NLL}}$ \\
\hline \multirow[t]{4}{*}{40.1 .2} & $\tilde{q} \tilde{\tilde{q}}$ & $1.41_{-0.24}^{+0.26} \times 10^{-3}$ & $1.68_{-0.14}^{+0.16} \times 10^{-3}$ & $\begin{array}{l}+10 \% \\
-9.7 \%\end{array}$ & 1.20 \\
\hline & $\tilde{q} \tilde{q}$ & $1.05_{-0.15}^{+0.13} \times 10^{-2}$ & $1.13_{-0.08}^{+0.09} \times 10^{-2}$ & & 1.07 \\
\hline & $\tilde{q} \tilde{g}$ & $4.46_{-0.73}^{+0.63} \times 10^{-3}$ & $5.74_{-0.67}^{+0.66} \times 10^{-3}$ & & 1.29 \\
\hline & $\tilde{g} \tilde{g}$ & $2.18_{-0.46}^{+0.46} \times 10^{-4}$ & $3.42_{-0.58}^{+0.63} \times 10^{-4}$ & & 1.57 \\
\hline \multirow[t]{4}{*}{40.1 .4} & $\tilde{q} \overline{\tilde{q}}$ & $2.28_{-0.41}^{+0.45} \times 10^{-4}$ & $2.83_{-0.24}^{+0.27} \times 10^{-4}$ & & 1.24 \\
\hline & $\tilde{q} \tilde{q}$ & $2.51_{-0.37}^{+0.35} \times 10^{-3}$ & $2.73_{-0.21}^{+0.23} \times 10^{-3}$ & $\begin{array}{l}+3.9 \% \\
-2.9 \%\end{array}$ & 1.09 \\
\hline & $\tilde{q} \tilde{g}$ & $7.25_{-1.28}^{+1.16} \times 10^{-4}$ & $9.71_{-1.21}^{+1.17} \times 10^{-4}$ & & 1.34 \\
\hline & $\tilde{g} \tilde{g}$ & $2.37_{-0.54}^{+0.57} \times 10^{-5}$ & $4.08_{-0.75}^{+0.81} \times 10^{-5}$ & & 1.72 \\
\hline \multirow[t]{4}{*}{40.2 .2} & $\tilde{q} \overline{\tilde{q}}$ & $1.34_{-0.23}^{+0.26} \times 10^{-3}$ & $1.60_{-0.13}^{+0.16} \times 10^{-3}$ & & 1.19 \\
\hline & $\tilde{q} \tilde{q}$ & $1.04_{-0.15}^{+0.14} \times 10^{-2}$ & $1.11_{-0.08}^{+0.10} \times 10^{-2}$ & & 1.07 \\
\hline & $\tilde{q} \tilde{g}$ & $6.74_{-1.11}^{+0.97} \times 10^{-3}$ & $8.65_{-0.98}^{+0.98} \times 10^{-3}$ & & 1.28 \\
\hline & $\tilde{g} \tilde{g}$ & $5.48_{-1.14}^{+1.15} \times 10^{-4}$ & $8.28_{-1.35}^{+1.46} \times 10^{-4}$ & $\begin{array}{l}+22 \% \\
-20 \%\end{array}$ & 1.51 \\
\hline \multirow[t]{4}{*}{40.2 .5} & $\tilde{q} \overline{\tilde{q}}$ & $6.14_{-1.17}^{+1.34} \times 10^{-5}$ & $7.75_{-0.67}^{+0.79} \times 10^{-5}$ & $\begin{array}{l}+15 \% \\
+15 \%\end{array}$ & 1.26 \\
\hline & $\tilde{q} \tilde{q}$ & $9.01_{-1.43}^{+1.41} \times 10^{-4}$ & $9.87_{-0.82}^{+0.94} \times 10^{-4}$ & & 1.10 \\
\hline & $\tilde{q} \tilde{g}$ & $3.64_{-0.67}^{+0.64} \times 10^{-4}$ & $4.98_{-0.62}^{+0.61} \times 10^{-4}$ & & 1.37 \\
\hline & $\tilde{g} \tilde{g}$ & $1.82_{-0.42}^{+0.46} \times 10^{-5}$ & $3.14_{-0.57}^{+0.62} \times 10^{-5}$ & & 1.72 \\
\hline \multirow[t]{4}{*}{40.3 .1} & $\tilde{q} \overline{\tilde{q}}$ & $1.00_{-0.17}^{+0.18} \times 10^{-3}$ & $1.19_{-0.10}^{+0.11} \times 10^{-3}$ & $\begin{array}{l}+11 \% \\
-10 \%\end{array}$ & 1.19 \\
\hline & $\tilde{q} \tilde{q}$ & $0.95_{-0.15}^{+0.15} \times 10^{-2}$ & $1.01_{-0.09}^{+0.12} \times 10^{-2}$ & $\begin{array}{l}+3.7 \% \\
-2.7 \%\end{array}$ & 1.06 \\
\hline & $\tilde{q} \tilde{g}$ & $2.83_{-0.49}^{+0.48} \times 10^{-2}$ & $3.57_{-0.35}^{+0.37} \times 10^{-2}$ & & 1.26 \\
\hline & $\tilde{g} \tilde{g}$ & $1.42_{-0.29}^{+0.31} \times 10^{-2}$ & $1.93_{-0.25}^{+0.27} \times 10^{-2}$ & & 1.36 \\
\hline \multirow[t]{4}{*}{40.3 .5} & $\tilde{q} \overline{\tilde{q}}$ & $7.05_{-1.30}^{+1.45} \times 10^{-5}$ & $8.81_{-0.75}^{+0.87} \times 10^{-5}$ & $\begin{array}{l}+15 \% \\
-15 \%\end{array}$ & 1.25 \\
\hline & $\tilde{q} \tilde{q}$ & $1.14_{-0.19}^{+0.20} \times 10^{-3}$ & $1.23_{-0.12}^{+0.15} \times 10^{-3}$ & $\begin{array}{l}+4.2 \% \\
-3.0 \%\end{array}$ & 1.08 \\
\hline & $\tilde{q} \tilde{g}$ & $2.87_{-0.53}^{+0.55} \times 10^{-3}$ & $3.78_{-0.40}^{+0.41} \times 10^{-3}$ & $\begin{array}{l}+10 \% \\
-9.8 \%\end{array}$ & 1.32 \\
\hline & $\tilde{g} \tilde{g}$ & $1.21_{-0.26}^{+0.29} \times 10^{-3}$ & $1.75_{-0.24}^{+0.26} \times 10^{-3}$ & $\begin{array}{l}+19 \% \\
-18 \%\end{array}$ & 1.45 \\
\hline \multirow[t]{4}{*}{ mGMSB2.2.1 } & $\tilde{q} \overline{\tilde{q}}$ & $1.48_{-0.25}^{+0.26} \times 10^{-3}$ & $1.76_{-0.14}^{+0.17} \times 10^{-3}$ & $\begin{array}{l}+10 \% \\
-9.6 \%\end{array}$ & 1.19 \\
\hline & $\tilde{q} \tilde{q}$ & $1.26_{-0.19}^{+0.19} \times 10^{-2}$ & $1.34_{-0.12}^{+0.15} \times 10^{-2}$ & & 1.06 \\
\hline & $\tilde{q} \tilde{g}$ & $2.43_{-0.41}^{+0.39} \times 10^{-2}$ & $3.07_{-0.31}^{+0.32} \times 10^{-2}$ & $\begin{array}{l}+8.1 \% \\
-7.6 \%\end{array}$ & 1.26 \\
\hline & $\tilde{g} \tilde{g}$ & $7.17_{-1.46}^{+1.54} \times 10^{-3}$ & $9.92_{-1.35}^{+1.45} \times 10^{-3}$ & $\begin{array}{l}+17 \% \\
-16 \%\end{array}$ & 1.38 \\
\hline \multirow[t]{4}{*}{ mGMSB2.2.4 } & $\tilde{q} \overline{\tilde{q}}$ & $1.04_{-0.19}^{+0.21} \times 10^{-4}$ & $1.29_{-0.11}^{+0.13} \times 10^{-4}$ & $\begin{array}{l}+14 \% \\
-14 \%\end{array}$ & 1.24 \\
\hline & $\tilde{q} \tilde{q}$ & $1.52_{-0.25}^{+0.26} \times 10^{-3}$ & $1.65_{-0.15}^{+0.19} \times 10^{-3}$ & $\begin{array}{l}+4.1 \% \\
-2.9 \%\end{array}$ & 1.08 \\
\hline & $\tilde{q} \tilde{g}$ & $2.67_{-0.49}^{+0.49} \times 10^{-3}$ & $3.52_{-0.38}^{+0.39} \times 10^{-3}$ & $\begin{array}{l}+10 \% \\
-9.9 \%\end{array}$ & 1.32 \\
\hline & $\tilde{g} \tilde{g}$ & $0.74_{-0.16}^{+0.18} \times 10^{-3}$ & $1.09_{-0.15}^{+0.17} \times 10^{-3}$ & $\begin{array}{l}+20 \% \\
-19 \%\end{array}$ & 1.47 \\
\hline
\end{tabular}

Table 6. NLL results for CMSSM benchmark points for $\tan \beta=40, A_{0}=-500$ and mGMSB benchmark points.

$25 \%$ range at the LHC with $\sqrt{s}=7 \mathrm{TeV}$ and at the $12-20 \%$-level at a centre-of-mass energy of $14 \mathrm{TeV}$. The NLL-corrections are therefore moderate for the mass-ranges accessible at the LHC, but larger than for light-flavour squark-antisquark production for the same masses (c.f. figure 7). Contrary to the latter, for stops the most substantial contribution is given 


\begin{tabular}{|c|l|l|l|c|}
\hline & \multicolumn{4}{|c|}{$\sigma\left(p p \rightarrow \tilde{t}_{1} \overline{\tilde{t}}_{1}\right)(\mathrm{pb}), \sqrt{s}=7 \mathrm{TeV}$} \\
\hline$m_{\tilde{t}_{1}}$ & $\mathrm{NLO}$ & $\mathrm{NLL}$ & $\Delta_{\mathrm{PDF}}$ & $K_{\mathrm{NLL}}$ \\
\hline 100 & $4.18_{-0.59}^{+0.63} \times 10^{2}$ & $4.77_{-0.66}^{+0.87} \times 10^{2}$ & ${ }_{-2.4 \%}^{+2.0 \%}$ & 1.14 \\
\hline 200 & $1.28_{-0.18}^{+0.17} \times 10^{1}$ & $1.47_{-0.19}^{+0.25} \times 10^{1}$ & ${ }_{-4.1 \%}^{+3.1 \%}$ & 1.15 \\
\hline 300 & $1.28_{-0.18}^{+0.17}$ & $1.49_{-0.19}^{+0.24}$ & ${ }_{-5.3 \%}^{+4.4 \%}$ & 1.17 \\
\hline 400 & $2.12_{-0.31}^{+0.28} \times 10^{-1}$ & $2.50_{-0.30}^{+0.38} \times 10^{-1}$ & ${ }_{-6.4 \%}^{+5.7 \%}$ & 1.18 \\
\hline
\end{tabular}

Table 7. Stop-antistop production cross sections. All masses are given in GeV.

by pure soft resummation, with Coulomb effects, including bound-state corrections, in the $5 \%$ range of the NLO result. As the partonic cross sections for the gluon-fusion channel are identical for the light-flavour and top squarks, the different behaviour can be attributed to the quark-antiquark induced channel. For the light-flavour squarks it is dominated by $S$-wave colour-singlet production with a large, attractive Coulomb potential. For stop production the colour-singlet channel is absent, and only the $P$-wave colour-octet channel with a smaller repulsive Coulomb potential contributes. The increased relative size of the soft corrections compared to the light-flavour squarks follows from the $P$-wave suppression of the quark-antiquark channel and the resulting dominance of the gluon-fusion channel with larger soft corrections due to the colour factors $C_{A}=N_{C}$.

The total theoretical uncertainty of the fixed-order NLO result and the resummed cross section, with and without Coulomb resummation, is compared in figure 14. The width of the uncertainty band for NLL and $\mathrm{NLL}_{s+h}$ is similar, consistent with the observed small effect of Coulomb resummation. One can also notice that the resummed results shows almost no uncertainty reduction compared to the NLO result, except for the highmass range.

\subsection{Comparison with Mellin-space results}

Results for NLL resummation of soft logarithms for squark and gluino production have been presented earlier in [14-18]. These works adopt the so-called Mellin-space formalism, in which the threshold logarithms are exponentiated in Mellin-moment space, where singular terms appear as logarithms of the Mellin-moment $N$, and the resummed cross section is then numerically inverted back to momentum-space. In this section we compare these earlier predictions to the momentum-space formalism adopted here, using the numerical code NLL-fast [53] to compute the Mellin-space resummed cross sections. Since NLL-fast provides results for soft-resummation only (i.e. no Coulomb effects beyond $\mathcal{O}\left(\alpha_{s}\right)$ are included), for the comparison we introduce two additional NLL implementations:

- $N L L_{s}$ : this implementation includes NLL resummation of soft logarithms, but no Coulomb or hard effects beyond $\mathcal{O}\left(\alpha_{s}\right)$. This is achieved using eqs. (2.19) and (2.24) and setting $\mu_{h}=\mu_{f}$. For the soft scale we adopt the running scale given in eqs. (2.28), (2.29). 


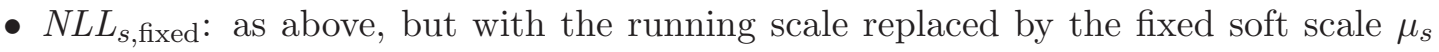
determined from eq. (2.27).

The $K$-factor for $\mathrm{NLL}_{s}$ (solid blue), $\mathrm{NLL}_{s, \text { fixed }}$ (dashed red) and the Mellin-space result (green dots) from NLL-fast are shown in figure 15. For clarity, we do not show the resummation uncertainties of the $\mathrm{NLL}_{s}$ and $\mathrm{NLL}_{s}$,fixed results that are significant, especially for smaller masses, as can be anticipated from the full NLL-results including Coulomb resummation in figure 6. Even though the difference between $\mathrm{NLL}_{s}$ and $\mathrm{NLL}_{\text {Mellin }}$ can be sizable, the envelope of the three curves in the plots in figure 15 is well within the theoretical uncertainties of the predictions, and is thus correctly accounted for by our estimate of the intrinsic ambiguities of NLL resummation.

The comparison of our default running-scale implementation $\mathrm{NLL}_{s}$ with $\mathrm{NLL}_{\mathrm{Mellin}}$ shows different features for the various production channels. For squark-antisquark and gluino-pair production the agreement is overall reasonable, although the behaviour as a function of the mass is different in both cases, leading to a better agreement at large masses in the former case and at smaller masses in the latter. For squark-gluino production, the agreement also improves at larger masses. In contrast, for the squark-squark production channel, there is a constant shift, and the differences are sizable over the whole mass range. Since the NLL corrections are small for this production channel, the discrepancies in the cross-section predictions are however less important. For all processes, the fixed-scale momentum-space results $\mathrm{NLL}_{s, \text { fixed }}$ are closer to the Mellin-space results, with similar magnitude and mass-dependence of the corrections. Since $\mathrm{NLL}_{s}$ and $\mathrm{NLL}_{\mathrm{Mellin}}$ both resum threshold logarithms ( $\ln \beta$ and $\ln N$ respectively) appearing in the partonic cross sections, while $\mathrm{NLL}_{s, \text { fixed }}$ resums logarithms in the hadronic cross section, this behaviour is somewhat counter-intuitive and deserves further studies in the future. ${ }^{8}$ The spread of the three predictions for stop production is comparable to the one observed for the other SUSY processes in the same mass range. In this case, however, the two momentum-space predictions with a fixed and running soft scale show a better agreement with each other.

It is interesting to note that the NNLL results for squark-antisquark production obtained in the $N$-space approach in [24] are very similar to our best prediction for this channel in figures 7 and 8. Part of this agreement can be attributed to the fact that ref. [24] includes the interference of the first Coulomb correction with higher-order soft corrections, which give a dominant contribution to our full NLL predictions (i.e. to the difference between the red and blue curves in figures 7 and 8), and to the difference between the NLL and NNLL results in [24]. However, our prediction includes higher-order Coulomb corrections and bound-state effects not included in the results of [24], while their results include one-loop hard corrections and NNLL soft corrections not included in ours. Therefore the good agreement is to some extent fortunate, and cannot necessarily be expected for the other processes where the Coulomb corrections are of less relative importance (in particular for squark-squark production), and where an NNLL analysis remains to be performed.

\footnotetext{
${ }^{8}$ An analytic comparison of momentum-space resummation with fixed soft scale to $N$-space resummation has appeared recently for Drell-Yan production [56], but no such investigation for the running scale with a lower cutoff has been performed yet.
} 

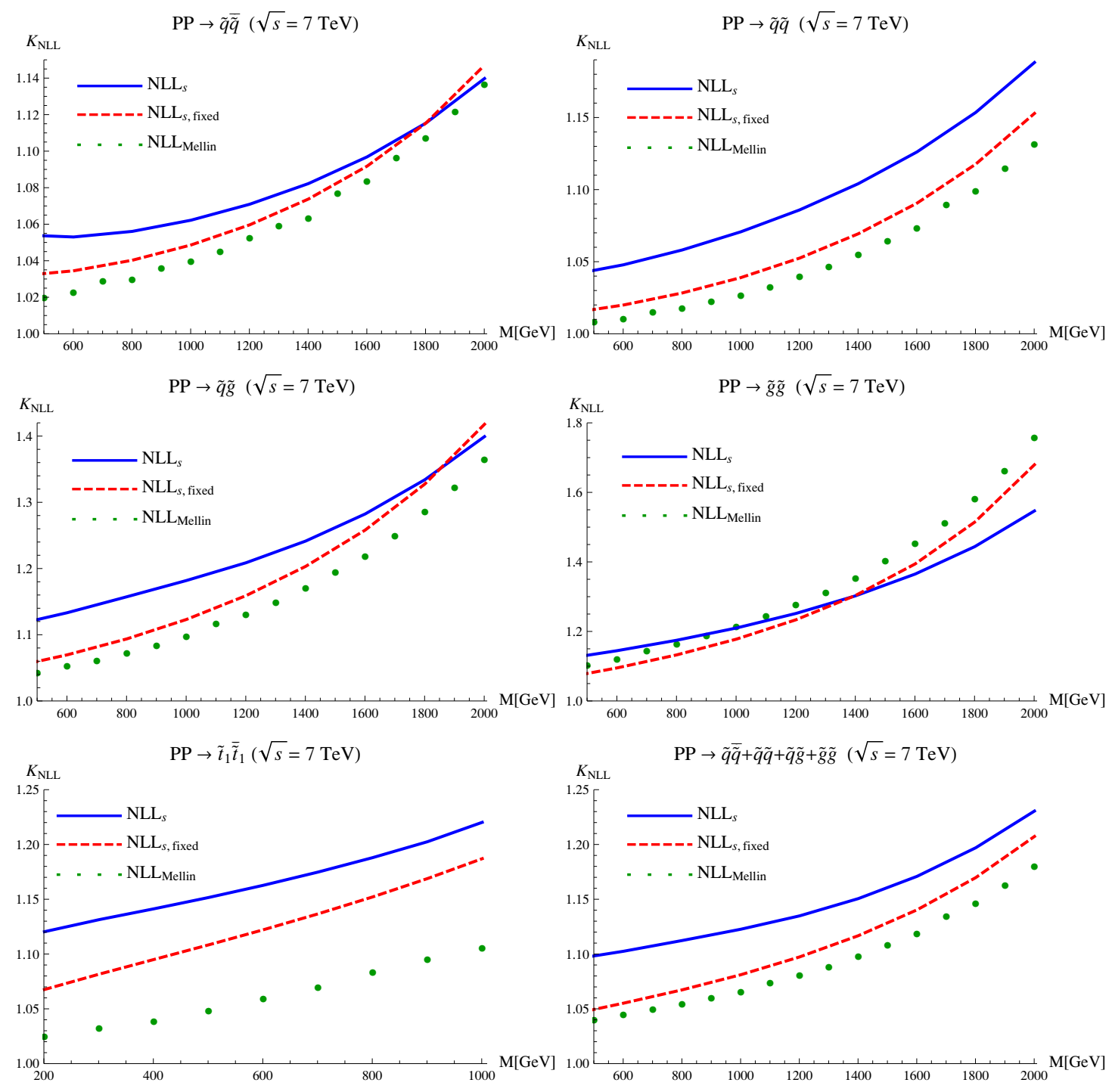

Figure 15. Comparison of soft resummation in the momentum-space formalism adopted in this work and in the conventional Mellin-space approach for squark-antisquark (top-left), squark-squark (top-right), squark-gluino (centre-left), gluino-gluino (centre-right), stop-antistop (bottom-left) production and the inclusive gluino and light-flavour squark cross section (bottom-right) at LHC with $\sqrt{s}=7 \mathrm{TeV}$. The plots show the $K$-factor for our default running-scale implementation $\left(\mathrm{NLL}_{s}\right.$, solid blue), for the fixed-scale implementation $\left(\mathrm{NLL}_{s}\right.$, fixed, dashed red) and for the Mellin-space result $\left(\mathrm{NLL}_{\mathrm{Mellin}}\right.$, green dots) obtained with NLL-fast. See the text for more details.

Furthermore, a combined NNLL soft/Coulomb resummation as performed for top-quark pair production in [32] would include higher-order Coulomb effects included neither in our present predictions nor in [24].

\section{Conclusions and outlook}

We have performed a combined resummation of soft-gluon and Coulomb effects for all squark- and gluino-pair production channels at the LHC, including bound-state effects, 
based on the formalism derived in $[30,31]$. We have also extended the derivation of the factorization and resummation formalism to the case of stop-antistop production with a quark-antiquark initial state, that proceeds through a $P$-wave. The corrections from higherorder Coulomb and bound-state effects and their interference with soft corrections are not included in previous predictions and can be sizeable, in particular for gluino-pair production, where they are as large as the soft-gluon corrections alone, and for squark-antisquark production where the effect is even bigger. For benchmark scenarios with moderate squarkgluino mass splitting the effect on the total inclusive squark and gluino production cross section is less pronounced but still relevant, with the total NLL corrections of the order of 15-30\% of the NLO cross section. Therefore these predictions should be taken into account in the analysis of the upcoming LHC results expected this year. To facilitate the application of our results we provided numerical predictions for some of the benchmark points defined in [36], and include grid files with our results for squark and gluino masses in the 200-2000 GeV (200-2500 GeV) range at the $7 \mathrm{TeV}(8 \mathrm{TeV})$ LHC with the arXiv submission of this paper.

Our results for soft-gluon resummation alone, obtained in the momentum-space resummation approach [33-35] and with the scale-setting procedure introduced in [32], agree within our estimate of resummation ambiguities with results obtained in the Mellin-space formalism $[16,17]$, although a more detailed study of the relation between the approaches and the different scale choices within the momentum space framework would be desirable.

In our analysis, the squarks and gluinos have been treated as stable, but contributions to the cross section from below the nominal production threshold have been included through bound-state corrections. This is expected to take the effect of small, but finite, widths to some extent into account. A more refined analysis is possible in our framework using a complex energy in the argument of the potential function in the factorization formula (2.13), as done in recent studies of the invariant mass spectrum of gluino-pair and squark-gluino production [25-27]. The investigation of the combined effect with soft resummation, as well as the extension to NNLL accuracy following ref. [32], is left for future work.

\section{Acknowledgments}

We would like to thank M. Beneke, W. Beenakker, A. Kulesza and I. Niessen for useful discussions and G. Watt for providing us with MSTW PDFs with improved accuracy in the evolution at large $x$. The work of P.F. is supported by the "Stichting voor Fundamenteel Onderzoek der Materie (FOM)", the work of C.W. by the research programme Mozaiek, which is partly financed by the Netherlands Organisation for Scientific Research (NWO).

\section{A NLL resummation for stop-antistop production}

In this appendix we derive the factorization formula (2.13) for stop-antistop production from quark-antiquark annihilation that proceeds in a $P$-wave state. While the aim is to 
establish resummation at NLL accuracy, the arguments suggest that the combined softCoulomb resummation can be performed at NNLL accuracy as well. Our reasoning applies to partonic subprocesses with a leading $P$-wave contribution, as relevant for stop-antistop production, but not to $P$-wave contributions to $S$-wave dominated subprocesses.

Following [31] the scattering process $q \bar{q} \rightarrow \tilde{t} \bar{t}+X$ is described in terms of an effective field theory using soft-collinear effective theory (SCET) [57-60] for the initial state quarks and potential non-relativistic QCD (pNRQCD) [61-64] for the stop and antistop. In this framework, the scattering amplitude in the full MSSM is expressed in terms of expectation values of EFT operators multiplied by short-distance coefficients $C$, that contain the dependence on hard modes not present in the EFT (e.g. gluinos, hard off-shell gluons):

$$
\mathcal{A}(q \bar{q} \rightarrow \tilde{t} \bar{t} X)=\sum_{\ell, R_{\alpha}} C^{(\ell), R_{\alpha}}(\mu)\left\langle\tilde{\tilde{t}} X_{s}\left|\mathcal{O}^{(\ell), R_{\alpha}}(\mu)\right| q \bar{q}\right\rangle_{\mathrm{EFT}} .
$$

The series in $\ell$ accounts for the threshold expansion in powers of $\beta$, while $R_{\alpha}=1,8$ are the irreducible colour representations of the stop-antistop pair. Due to the threshold kinematics, $\left(k_{1}+k_{2}\right)^{2} \sim 4 M^{2}$, no collinear modes can appear in the final state $X_{s}$, that only contains soft modes. Both the short-distance coefficients $C^{(\ell), R_{\alpha}}$ and the operators $\mathcal{O}^{(\ell), R_{\alpha}}$ can carry open Lorentz and spin indices, that have been suppressed. For NLL resummation, only the colour-octet state is relevant. ${ }^{9}$ For the case of $P$-wave production, the leading $\ell=0$ term in the expansion of the scattering amplitude (A.1) vanishes and the first non-vanishing term occurs for $\ell=1$, i.e. it is suppressed by $\mathcal{O}(\beta)$ compared to the $S$-wave case. The $P$-wave production operator for a colour-octet state reads

$$
\mathcal{O}_{\mu}^{(1), 8}(\mu)=\frac{1}{\sqrt{2}}\left[\left(\bar{\xi}_{\bar{c}} W_{\bar{c}}\right) T^{a}\left(W_{c}^{\dagger} \xi_{c}\right)\right]\left[\psi^{\dagger} T^{a}\left(-\frac{i}{2} \overleftrightarrow{D}_{\mu, \top}\right) \chi\right](\mu)
$$

Here the field $\psi^{\dagger}(\chi)$ is a non-relativistic field that creates the stop (antistop) while $\xi_{c}$ $\left(\bar{\xi}_{\bar{c}}\right)$ is a collinear (anti-collinear) field that destroys the initial-state quark with momentum $\sim \sqrt{\hat{s}} / 2 n$ (antiquark with momentum $\sim \sqrt{\hat{s}} / 2 \bar{n}$ ), with light-cone vectors $n^{2}=\bar{n}^{2}=0$, $n \cdot \bar{n}=2$. The $W_{c}\left(W_{\bar{c}}\right)$ are collinear (anti-collinear) Wilson lines summing up collinear gluon emission to all orders $[57,58]$. $D_{\top}$ denotes the projection of the covariant derivative $D_{\mu}=\left(\partial_{\mu}-i g_{s} T^{a} A_{\mu}^{a}(x)\right)$ in the direction orthogonal to the timelike velocity $w^{\mu}$ of the stop-antistop pair.

At this stage, the operator (A.2) is written in NRQCD and contains the full gluon field $A$. This does not yet incorporate a systematic expansion in $\beta$. For this purpose we adopt potential non-relativistic QCD (pNRQCD) [61-64], where only soft gluons with momenta scaling as

$$
q \sim M \beta^{2}
$$

and the non-relativistic fields with so-called potential momenta scaling as

$$
k_{0} \sim M \beta^{2}, \quad \vec{k} \sim M \beta
$$

\footnotetext{
${ }^{9}$ At NLO a finite, non-logarithmic colour-singlet contribution is generated by real-gluon emission [48] that is suppressed by $\alpha_{s} \beta^{2}$ compared to the leading $P$-wave contribution, and therefore not even relevant at NNLL. Since there is no leading $S$-wave contribution to $q \bar{q} \rightarrow \tilde{t} \tilde{t}$, the situation is simpler than for quarkonium production, where singularities cancel among colour octet $S$-wave and singlet $P$-wave channels [65].
} 
are retained in the EFT, while potential gluons with momenta scaling like (A.4) and modes with momenta of order $M \beta$ are integrated out. After this step, the covariant derivative in (A.2) contains only the soft gluon field $A_{s}$ with the same scaling as the soft momentum,

$$
A_{s}^{\mu} \sim M \beta^{2}
$$

Therefore the term $g_{s} A_{s, \mu} \top$ in the covariant derivative is suppressed by a factor $g_{s} \beta$ compared to the derivative term $\partial_{\mu \top} \sim \beta$ and the production operator (A.2) at leading power in pNRQCD is simply given by

$$
\mathcal{O}_{\mu}^{(1), 8}(\mu)=\frac{1}{\sqrt{2}}\left[\left(\bar{\xi}_{\bar{c}} W_{\bar{c}}\right) T^{a}\left(W_{c}^{\dagger} \xi_{c}\right)\right]\left[\psi^{\dagger} T^{a}\left(-\frac{i}{2} \overleftrightarrow{\partial}_{\mu, \top}\right) \chi\right](\mu)
$$

The potential, collinear and anti-collinear fields interact only through the exchange of soft gluons. These interactions can be removed from the leading effective-theory Lagrangians by a decoupling transformation of the collinear $[58,66]$ and potential [31] fields involving soft Wilson lines of the schematic form $W_{c}^{\dagger} \xi_{c}=S_{n} W_{c}^{(0) \dagger} \xi_{c}^{(0)}$ and $\chi=S_{w} \chi^{(0)}$, and similar transformations for the conjugate fields. For definitions of the Wilson lines $S_{n}$ and $S_{w}$ see [31]. Since the fields with superscript (0) do not interact with each other in the leading effective Lagrangians, the scattering amplitude assumes a factorized form:

$$
\begin{aligned}
\mathcal{A}\left(q \bar{q} \rightarrow \tilde{t}_{i} \overline{\tilde{t}}_{i} X\right)= & \left(-\frac{i}{2 \sqrt{2}}\right) C_{\mu}^{(1), 8}(\mu)\left\langle 0\left|\left(W_{c}^{(0) \dagger} \xi_{c}^{(0)}\right)_{j_{1}}\right| q\right\rangle\left\langle 0\left|\left(\bar{\xi}_{\bar{c}} W_{\bar{c}}\right)_{j_{2}}\right| \bar{q}\right\rangle \\
& \times\left\langle X_{s}\left|\left(S_{\bar{n}}^{\dagger} T^{a} S_{n}\right)_{j_{2} j_{1}}\left(S_{w}^{\dagger} T^{a} S_{w}\right)_{j_{3} j_{4}}\right| 0\right\rangle\left\langle\tilde{t}_{i} \overline{\tilde{t}}_{i}\left|\psi_{j_{3}}^{(0) \dagger} \overleftrightarrow{\partial_{\top}^{\mu}} \chi_{j_{4}}^{(0)}\right| 0\right\rangle
\end{aligned}
$$

Note that the factorization (A.7) holds only at leading power in the $\beta$-expansion, since the field redefinition does not remove the interaction with the spatial components of the soft gluons in the $P$-wave production operators, or in the subleading effective Lagrangians. We will argue below that these corrections are not relevant at NLL accuracy for stop-antistop production from quark-antiquark annihilation.

Using the representation of the scattering amplitude (A.7) and following the steps discussed in detail in [31] one derives the factorization formula

$$
\hat{\sigma}_{q \bar{q}}(\hat{s}, \mu)=H_{q \bar{q}}^{8}(\mu) \int d \omega J_{8}^{P}\left(E-\frac{\omega}{2}\right) W^{8}(\omega, \mu) .
$$

Here the potential function for $P$-wave production in a colour octet state is given by

$$
J_{8}^{P}(q)=\frac{1}{2} \int d^{4} z e^{i q \cdot z}\left\langle 0\left|\left[\chi^{(0) \dagger} \overleftrightarrow{\partial_{\top}^{\mu}} T^{a} \psi^{(0)}\right](z)\left[\psi^{(0) \dagger} T^{a} \overleftrightarrow{\partial \uparrow}, \mu \chi^{(0)}\right](0)\right| 0\right\rangle
$$

while the colour-octet soft function (defined in terms of the squared matrix element of the soft Wilson lines in (A.7)) is identical to that appearing for $S$-wave production [30]. The factorization formula resembles that for $S$-wave production, up to the replacement of the potential function by the appropriate $P$-wave expression.

We now show that no corrections to (A.8) appear at NNLL. As far as soft-gluon effects are concerned, we have argued that the corrections to the production operator (A.6) from 


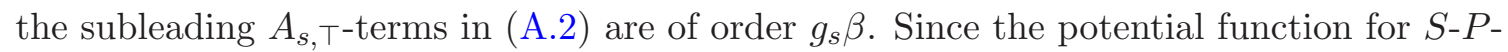
wave interference vanishes, there can be no contributions to the total cross section where

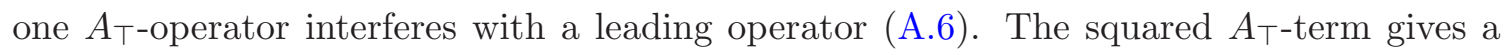
correction to the cross section of the order ${ }^{10} \Delta \sigma \sim \sigma^{0} \alpha_{s} \beta^{2}\{1, \ln \beta\}$. These corrections are at most of order $\mathrm{N}^{3} \mathrm{LL}$ in the combined counting $\alpha_{s} / \beta \sim 1$ and $\alpha_{s} \ln \beta \sim 1$.

Following [31] one sees that insertions of higher-order soft-collinear interactions into the matrix element (A.1) are at least suppressed by $\mathcal{O}\left(\beta^{2}\right)$ and therefore are beyond NNLL. For $P$-wave production it has to be shown in addition that no contributions to the $\ell=1$ operators in (A.1) are generated from partonic channels with leading $S$-wave $(\ell=0)$ contributions. In the EFT language they would correspond to operators where one or both of the quark/antiquark fields are replaced by (anti)collinear gluon fields. A nonvanishing matrix element of such an operator in the $|\bar{q} q\rangle$ initial state requires splittings of a collinear quark into a collinear gluon and a soft quark that are mediated by $\mathcal{O}(\beta)$ suppressed interactions in the SCET Lagrangian [60] (recall that no collinear final state particles appear at partonic threshold). Since there is no $q g$-initiated production of stopantistop pairs at leading order, two subleading splittings are required so these contributions to the amplitude are suppressed by $g_{s}^{2} \beta$ compared to the $\ell=1$ term. The resulting real corrections to the total cross section are therefore even further suppressed compared to the subleading soft effects.

Finally, subleading soft-potential effects due to chromoelectric $\vec{x} \cdot \vec{E}_{s}$ interactions [64] have been shown to be beyond NNLL in the $S$-wave case [31] since the potential function with a single insertion of an operator $\sim \vec{x}$ vanishes by rotational invariance. The same argument holds for the $P$-wave potential function. ${ }^{11}$ Furthermore, as mentioned before, due to the absence of a leading $S$-wave contribution to the quark-antiquark channel, no mixing of $S$-wave and $P$-wave states by subleading interactions appears. Therefore, while we didn't perform an exhaustive study of effects beyond NLL, we do not encounter an obstruction for NNLL resummation for the quark-antiquark initial-state contribution to stop-antistop production. Note, however, that Coulomb resummation at this accuracy requires the computation of the NLO $P$-wave Coulomb Green's function for scalar particles.

\section{B Resummation functions}

The resummation functions appearing in (2.18) are given by

$$
\begin{gathered}
U_{i}\left(M, \mu_{h}, \mu_{f}, \mu_{s}\right)=\left(\frac{4 M^{2}}{\mu_{h}^{2}}\right)^{-2 a_{\Gamma}\left(\mu_{h}, \mu_{s}\right)}\left(\frac{\mu_{h}^{2}}{\mu_{s}^{2}}\right)^{\eta} \times \exp \left[4\left(S\left(\mu_{h}, \mu_{f}\right)-S\left(\mu_{s}, \mu_{f}\right)\right)\right. \\
\left.-2 a_{i}^{V}\left(\mu_{h}, \mu_{s}\right)+2 a^{\phi, r}\left(\mu_{s}, \mu_{f}\right)+2 a^{\phi, r^{\prime}}\left(\mu_{s}, \mu_{f}\right)\right]
\end{gathered}
$$

\footnotetext{
${ }^{10}$ We have allowed for logarithmic corrections in the generalized potential function from contractions of the two gluon insertions. A simple estimate indicates, however, that these are in fact absent.

${ }^{11} \mathrm{~A}$ possible interference of a single $A_{T}$-term in the production operator with a chromoelectric vertex might lead to a non-vanishing potential function but would be of the same order $\alpha_{s} \beta^{2}\{1, \ln \beta\}$ as the soft contributions considered above.
} 
and $\eta=2 a_{\Gamma}\left(\mu_{s}, \mu_{f}\right)$. The labels $r, r^{\prime}$ denote the colour representation of the initial-state partons $p, p^{\prime}$. At NLL the functions $S, a_{i}^{V}$ and $a_{\Gamma}$ are given by $[31,35]$

$$
\begin{gathered}
S\left(\mu_{i}, \mu_{j}\right)=\frac{C_{r}+C_{r^{\prime}}}{2 \beta_{0}^{2}}\left[\frac{4 \pi}{\alpha_{s}\left(\mu_{i}\right)}\left(1-\frac{1}{r}-\ln r\right)+\left(2 K-\frac{\beta_{1}}{\beta_{0}}\right)(1-r+\ln r)+\frac{\beta_{1}}{2 \beta_{0}} \ln ^{2} r\right], \\
a_{\Gamma}\left(\mu_{i}, \mu_{j}\right)=\frac{C_{r}+C_{r}}{\beta_{0}} \ln r, \quad a_{i}^{V}\left(\mu_{i}, \mu_{j}\right)=\frac{\gamma_{i}^{(0), V}}{2 \beta_{0}} \ln r, \quad a^{\phi, r}\left(\mu_{i}, \mu_{j}\right)=\frac{\gamma^{(0) \phi, r}}{2 \beta_{0}} \ln r,
\end{gathered}
$$

where $r=\alpha_{s}\left(\mu_{j}\right) / \alpha_{s}\left(\mu_{i}\right)$ and $\gamma_{i}^{V}=\gamma^{r}+\gamma^{r^{\prime}}+\gamma_{H, s}^{R_{\alpha}}$. The one-loop anomalous-dimension coefficients appearing here are given by

$$
\gamma_{H, s}^{(0), R_{\alpha}}=-2 C_{R_{\alpha}}, \quad \gamma^{(0) 3}=-3 C_{F}=-\gamma^{(0) \phi, 3}, \quad \gamma^{(0) 8}=-\beta_{0}=-\gamma^{(0) \phi, 8}
$$

and the coefficients of the beta function are

$$
\beta_{0}=\frac{11}{3} C_{A}-\frac{2}{3} n_{f}, \quad \beta_{1}=\frac{34}{3} C_{A}^{2}-\frac{10}{3} C_{A} n_{f}-2 C_{F} n_{f} .
$$

We also used the factor $K=\left(\frac{67}{18}-\frac{\pi^{2}}{6}\right) C_{A}-\frac{10}{9} T_{F} n_{f}$ appearing in the ratio of one- and two-loop cusp anomalous dimensions. The explicit values of the Casimir invariants for the $\mathrm{SU}(3)$ representations relevant for squark and gluino production are:

$$
C_{1}=0, \quad C_{3}=\frac{4}{3}, \quad C_{6}=\frac{10}{3}, \quad C_{8}=3, \quad C_{10}=6, \quad C_{15}=\frac{16}{3}, \quad C_{27}=8 .
$$

\section{Determination of $\mu_{s}$ and $\beta_{\text {cut }}$}

The soft scale $\mu_{s}$ used in the fixed-scale implementation, NLL fixed, is defined by eq. (2.27). For equal squark and gluino masses, $m_{\tilde{q}}=m_{\tilde{g}}$, the minimization procedure yields the following results:

$$
\begin{array}{lll}
\tilde{q} \bar{q}: & \mu_{s}=127-170 \mathrm{GeV}(\sqrt{s}=7 \mathrm{TeV}), & \mu_{s}=146-376 \mathrm{GeV}(\sqrt{s}=14 \mathrm{TeV}), \\
\tilde{q} \tilde{q}: & \mu_{s}=122-135 \mathrm{GeV}(\sqrt{s}=7 \mathrm{TeV}), & \mu_{s}=146-312 \mathrm{GeV}(\sqrt{s}=14 \mathrm{TeV}), \\
\tilde{g} \tilde{q}: & \mu_{s}=109-141 \mathrm{GeV}(\sqrt{s}=7 \mathrm{TeV}), & \mu_{s}=131-310 \mathrm{GeV}(\sqrt{s}=14 \mathrm{TeV}), \\
\tilde{g} \tilde{g}: & \mu_{s}=106-141 \mathrm{GeV}(\sqrt{s}=7 \mathrm{TeV}), & \mu_{s}=127-308 \mathrm{GeV}(\sqrt{s}=14 \mathrm{TeV}) .
\end{array}
$$

The scales for a centre-of-mass energy of $7 \mathrm{TeV}$ refers to a mass range $M=500-2000 \mathrm{GeV}$, while for a $14 \mathrm{TeV}$ LHC the mass interval $M=500-3000 \mathrm{GeV}$ was considered. For stopantistop production we obtain the scales

$$
\tilde{t \tilde{t}}: \quad \mu_{s}=29-138 \mathrm{GeV}(\sqrt{s}=7 \mathrm{TeV}), \quad \mu_{s}=31-230 \mathrm{GeV}(\sqrt{s}=14 \mathrm{TeV})
$$

for the mass range $m_{\tilde{t}}=100-1000 \mathrm{GeV}$ for a centre-of-mass energy of $7 \mathrm{TeV}$ and $m_{\tilde{t}}=100-$ $1400 \mathrm{GeV}$ at $14 \mathrm{TeV}$. In [35] it was suggested to fit the mass and energy dependence of the soft scales (C.1) by a function of the form

$$
\mu_{s}=\frac{M(1-\rho)}{\sqrt{a+b \rho}}
$$




\begin{tabular}{|c|c|c|}
\hline & LHC(7 TeV) & LHC $(14 \mathrm{TeV})$ \\
\hline$\tilde{q} \tilde{\tilde{q}}$ & $a=12.1328$ & $a=11.5289$ \\
& $b=159.828$ & $b=171.856$ \\
\hline$\tilde{q} \tilde{q}$ & $a=10.9627$ & $a=10.9981$ \\
& $b=265.414$ & $b=278.062$ \\
\hline$\tilde{g} \tilde{q}$ & $a=16.5459$ & $a=15.3779$ \\
& $b=235.227$ & $b=267.763$ \\
\hline$\tilde{g} \tilde{g}$ & $a=17.95$ & $a=16.7483$ \\
& $b=231.545$ & $b=264.702$ \\
\hline$\tilde{t} \tilde{t}$ & $a=16.8468$ & $a=15.7282$ \\
& $b=365.735$ & $b=481.045$ \\
\hline
\end{tabular}

Table 8. Coefficients of the fit (C.3) for the squark-gluino production processes at centre-of-mass energies of 7 and $14 \mathrm{TeV}$.

with $\rho=4 M^{2} / s$. The coefficients $a$ and $b$ for the different processes are given in table 8 , and provide a fit to (C.1) with accuracy better than $5 \%$ for the mass range considered.

The procedure used to determine $\beta_{\text {cut }}$, which enters the definition of the soft scale used in our default NLL implementation, eqs. (2.28) and (2.29), was explained in detail in [32]. Following [32] we introduce eight different cross sections

$$
\hat{\sigma}_{p p^{\prime}}\left(A_{<}, B_{>}, \beta_{\text {cut }}\right)=\hat{\sigma}_{p p^{\prime}}^{A_{<}} \theta\left(\beta_{\text {cut }}-\beta\right)+\hat{\sigma}_{p p^{\prime}}^{B} \theta\left(\beta-\beta_{\text {cut }}\right)
$$

defined using one of two possible matching prescriptions for the lower interval $\left(A_{<} \in\right.$ $\left.\left\{\mathrm{NLL}_{1}, \mathrm{NLL}_{2}\right\}\right)$ and one of four possible approximations for the upper interval $\left(B_{>} \in\right.$ $\left.\left\{\mathrm{NLL}_{2}, \mathrm{NLO}_{\mathrm{app}}, \mathrm{NNLO}_{\mathrm{A}}, \mathrm{NNLO}_{\mathrm{B}}\right\}\right)$. Here $\mathrm{NLL}_{2}$ denotes our default approximation, eq. (2.20), while $\mathrm{NLL}_{1}$ is the resummed result matched to the Born instead of the NLO cross section,

$$
\hat{\sigma}_{p p^{\prime}}^{\mathrm{NLL}_{1}}(\hat{s})=\left[\hat{\sigma}_{p p^{\prime}}^{\mathrm{NLL}}(\hat{s})-\hat{\sigma}_{p p^{\prime}}^{\mathrm{NLL}(0)}(\hat{s})\right]+\hat{\sigma}_{p p^{\prime}}^{\mathrm{LO}}(\hat{s}) .
$$

$\mathrm{NLO}_{\text {app }}$ represents the sum of the full Born cross section and the approximated NLO corrections given in (2.10), while the two NNLO approximations contain in addition the $O\left(\alpha_{s}^{4}\right)$ terms arising from the expansion of the NLL resummed result, including all of them $\left(\mathrm{NNLO}_{\mathrm{A}}\right)$ or only the subset which is completely determined at NLL $\left(\mathrm{NNLO}_{\mathrm{B}}\right)$. $\beta_{\text {cut }}$ is then determined such that the width of the envelope of the eight different implementations $\hat{\sigma}_{p p^{\prime}}\left(A_{<}, B_{>}, \beta_{\text {cut }}\right)$ is minimal. ${ }^{12}$

\footnotetext{
${ }^{12}$ The values (C.6) are also used for the $\mathrm{NLL}_{s+h}$ approximation (2.19), instead of recalculating $\beta_{\text {cut }}$ using that approximation in (C.4). Since the difference of NLL and $\mathrm{NLL}_{s+h}$ is used in order to assess the effect of Coulomb resummation, we consider it more meaningful to use the same $\beta_{\text {cut }}$ for both in order not to obscure the genuine Coulomb effects by different scale choices.
} 

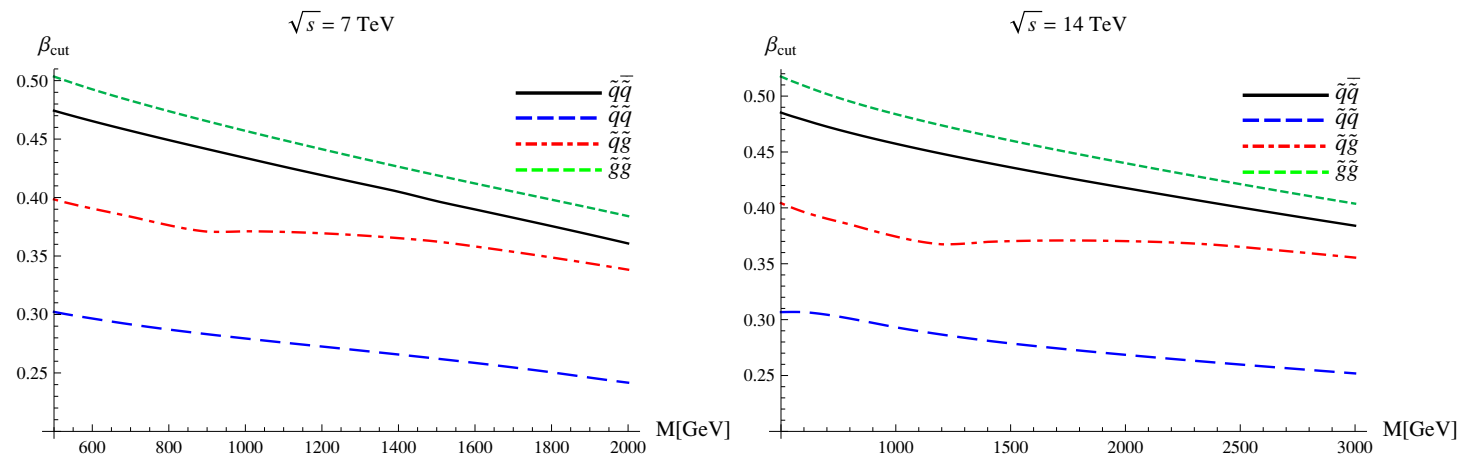

Figure 16. Mass dependence of the parameter $\beta_{\text {cut }}$ for the four SUSY production processes.

The procedure described provides, for the default choice $k_{s}=1$ and equal squark and gluino masses, the following values for $\beta_{\text {cut }}$ :

$$
\begin{aligned}
& \tilde{q} \overline{\tilde{q}}: \quad \beta_{\text {cut }}^{(7 \mathrm{TeV})}=0.47-0.36, \quad \beta_{\text {cut }}^{(8 \mathrm{TeV})}=0.48-0.34, \quad \beta_{\text {cut }}^{(14 \mathrm{TeV})}=0.48-0.38, \\
& \tilde{q} \tilde{q}: \quad \beta_{\text {cut }}^{(7 \mathrm{TeV})}=0.30-0.24, \quad \beta_{\text {cut }}^{(8 \mathrm{TeV})}=0.31-0.24, \quad \beta_{\text {cut }}^{(14 \mathrm{TeV})}=0.31-0.25, \\
& \tilde{g} \tilde{q}: \quad \beta_{\text {cut }}^{(7 \mathrm{TeV})}=0.40-0.34, \quad \beta_{\text {cut }}^{(8 \mathrm{TeV})}=0.40-0.33, \quad \beta_{\text {cut }}^{(14 \mathrm{TeV})}=0.40-0.36, \\
& \tilde{g} \tilde{g}: \quad \beta_{\text {cut }}^{(7 \mathrm{TeV})}=0.50-0.39, \quad \beta_{\text {cut }}^{(8 \mathrm{TeV})}=0.51-0.37, \quad \beta_{\text {cut }}^{(14 \mathrm{TeV})}=0.52-0.40 .
\end{aligned}
$$

The numbers refer to the usual mass range of $500-2000 \mathrm{GeV}$ at $\sqrt{s}=7 \mathrm{TeV}$ and $500-$ $3000 \mathrm{GeV}$ at $\sqrt{s}=14 \mathrm{TeV}$, and the exact mass dependence is plotted in figure 16 . We have also included the results for the mass range of $500-2500 \mathrm{GeV}$ at $\sqrt{s}=8 \mathrm{TeV}$. For stop-antistop production the result is

$$
\tilde{\tilde{t}}: \quad \beta_{\text {cut }}^{(7 \mathrm{TeV})}=0.53-0.40, \quad \beta_{\text {cut }}^{(8 \mathrm{TeV})}=0.53-0.39, \quad \beta_{\text {cut }}^{(14 \mathrm{TeV})}=0.54-0.41,
$$

for the mass range $m_{\tilde{t}}=100-1000 \mathrm{GeV}$ at $7 \mathrm{TeV}, m_{\tilde{t}}=100-1200 \mathrm{GeV}$ at $8 \mathrm{TeV}$ and $m_{\tilde{t}}=$ $100-1400 \mathrm{GeV}$ at $14 \mathrm{TeV}$. The theoretical ambiguities of the resummed result with the running scale are estimated as follows: i) the default implementation $\mathrm{NLL}_{2}$ is parametrized in terms of $\hat{E}$ instead of $\beta$, ii) the $\mathrm{NLL}_{2}$ result is computed for the values $k_{s}=1 / 2,2$, recomputing $\beta_{\text {cut }}$ anew for each choice and iii), the envelope of the eight cross sections (C.4) is taken while $\beta_{\text {cut }}$ is varied by $\pm 20 \%$. The uncertainties from the three sources are added in quadrature, and iii) gives the dominant contribution, in the $3-13 \%$ range depending on the process, while i) and ii) are usually below $2 \%$.

Open Access. This article is distributed under the terms of the Creative Commons Attribution License which permits any use, distribution and reproduction in any medium, provided the original author(s) and source are credited.

\section{References}

[1] X.P. Bueso, Supersymmetry searches at the Tevatron and the LHC collider experiments, arXiv:1112.1723 [INSPIRE]. 
[2] ATLAS collaboration, G. Aad et al., Search for squarks and gluinos using final states with jets and missing transverse momentum with the ATLAS detector in $\sqrt{s}=7 \mathrm{TeV}$ proton-proton collisions, Phys. Lett. B 710 (2012) 67 [arXiv:1109.6572] [INSPIRE].

[3] CMS collaboration, S. Chatrchyan et al., Search for supersymmetry at the LHC in events with jets and missing transverse energy, Phys. Rev. Lett. 107 (2011) 221804 [arXiv: 1109.2352] [INSPIRE].

[4] H. Baer, V. Barger, A. Lessa and X. Tata, Supersymmetry discovery potential of the LHC at $\sqrt{s}=10 \mathrm{TeV}$ and $14 \mathrm{TeV}$ without and with missing E(T), JHEP 09 (2009) 063 [arXiv:0907.1922] [INSPIRE].

[5] W. Beenakker, R. Hopker, M. Spira and P. Zerwas, Squark and gluino production at hadron colliders, Nucl. Phys. B 492 (1997) 51 [hep-ph/9610490] [inSPIRE].

[6] W. Beenakker, R. Hopker and M. Spira, PROSPINO: a program for the production of supersymmetric particles in next-to-leading order QCD, hep-ph/9611232 [INSPIRE].

[7] W. Beenakker, M. Krämer, T. Plehn, M. Spira and P. Zerwas, Stop production at hadron colliders, Nucl. Phys. B 515 (1998) 3 [hep-ph/9710451] [INSPIRE].

[8] S. Bornhauser, M. Drees, H.K. Dreiner and J.S. Kim, Electroweak contributions to squark pair production at the LHC, Phys. Rev. D 76 (2007) 095020 [arXiv:0709.2544] [INSPIRE].

[9] W. Hollik, M. Kollar and M.K. Trenkel, Hadronic production of top-squark pairs with electroweak NLO contributions, JHEP 02 (2008) 018 [arXiv:0712.0287] [INSPIRE].

[10] W. Hollik and E. Mirabella, Squark anti-squark pair production at the LHC: the electroweak contribution, JHEP 12 (2008) 087 [arXiv:0806.1433] [INSPIRE].

[11] W. Hollik, E. Mirabella and M.K. Trenkel, Electroweak contributions to squark-gluino production at the LHC, JHEP 02 (2009) 002 [arXiv:0810.1044] [INSPIRE].

[12] E. Mirabella, NLO electroweak contributions to gluino pair production at hadron colliders, JHEP 12 (2009) 012 [arXiv:0908.3318] [INSPIRE].

[13] J. Germer, W. Hollik, E. Mirabella and M.K. Trenkel, Hadronic production of squark-squark pairs: the electroweak contributions, JHEP 08 (2010) 023 [arXiv: 1004.2621] [INSPIRE].

[14] A. Kulesza and L. Motyka, Threshold resummation for squark-antisquark and gluino-pair production at the LHC, Phys. Rev. Lett. 102 (2009) 111802 [arXiv:0807.2405] [INSPIRE].

[15] A. Kulesza and L. Motyka, Soft gluon resummation for the production of gluino-gluino and squark-antisquark pairs at the LHC, Phys. Rev. D 80 (2009) 095004 [arXiv:0905.4749] [INSPIRE].

[16] W. Beenakker et al., Soft-gluon resummation for squark and gluino hadroproduction, JHEP 12 (2009) 041 [arXiv:0909.4418] [INSPIRE].

[17] W. Beenakker et al., Supersymmetric top and bottom squark production at hadron colliders, JHEP 08 (2010) 098 [arXiv: 1006.4771] [INSPIRE].

[18] W. Beenakker et al., Squark and gluino hadroproduction, Int. J. Mod. Phys. A 26 (2011) 2637 [arXiv:1105.1110] [InSPIRE].

[19] G.F. Sterman, Summation of large corrections to short distance hadronic cross-sections, Nucl. Phys. B 281 (1987) 310 [InSPIRE].

[20] S. Catani and L. Trentadue, Resummation of the QCD perturbative series for hard processes, Nucl. Phys. B 327 (1989) 323 [inSPIRE]. 
[21] N. Kidonakis and G.F. Sterman, Resummation for QCD hard scattering, Nucl. Phys. B 505 (1997) 321 [hep-ph/9705234] [INSPIRE].

[22] R. Bonciani, S. Catani, M.L. Mangano and P. Nason, NLL resummation of the heavy quark hadroproduction cross-section, Nucl. Phys. B 529 (1998) 424 [Erratum ibid. B 803 (2008) 234] [hep-ph/9801375] [INSPIRE].

[23] M. Czakon, A. Mitov and G.F. Sterman, Threshold resummation for top-pair hadroproduction to next-to-next-to-leading log, Phys. Rev. D 80 (2009) 074017 [arXiv:0907.1790] [INSPIRE].

[24] W. Beenakker et al., NNLL resummation for squark-antisquark pair production at the LHC, JHEP 01 (2012) 076 [arXiv:1110.2446] [INSPIRE].

[25] K. Hagiwara and H. Yokoya, Bound-state effects on gluino-pair production at hadron colliders, JHEP 10 (2009) 049 [arXiv:0909.3204] [INSPIRE].

[26] M.R. Kauth, J.H. Kuhn, P. Marquard and M. Steinhauser, Gluino pair production at the LHC: the threshold, Nucl. Phys. B $\mathbf{8 5 7}$ (2012) 28 [arXiv:1108.0361] [INSPIRE].

[27] M.R. Kauth, A. Kress and J.H. Kuhn, Gluino-squark production at the LHC: the threshold, JHEP 12 (2011) 104 [arXiv:1108.0542] [INSPIRE].

[28] U. Langenfeld and S.-O. Moch, Higher-order soft corrections to squark hadro-production, Phys. Lett. B 675 (2009) 210 [arXiv:0901.0802] [INSPIRE].

[29] U. Langenfeld, Threshold improved QCD corrections for stop-antistop production at hadron colliders, JHEP 07 (2011) 052 [arXiv: 1011.3341] [INSPIRE].

[30] M. Beneke, P. Falgari and C. Schwinn, Soft radiation in heavy-particle pair production: all-order colour structure and two-loop anomalous dimension, Nucl. Phys. B $\mathbf{8 2 8}$ (2010) 69 [arXiv: 0907.1443] [INSPIRE].

[31] M. Beneke, P. Falgari and C. Schwinn, Threshold resummation for pair production of coloured heavy (s)particles at hadron colliders, Nucl. Phys. B 842 (2011) 414 [arXiv: 1007.5414] [INSPIRE].

[32] M. Beneke, P. Falgari, S. Klein and C. Schwinn, Hadronic top-quark pair production with NNLL threshold resummation, Nucl. Phys. B 855 (2012) 695 [arXiv:1109.1536] [INSPIRE].

[33] T. Becher and M. Neubert, Threshold resummation in momentum space from effective field theory, Phys. Rev. Lett. 97 (2006) 082001 [hep-ph/0605050] [INSPIRE].

[34] T. Becher, M. Neubert and B.D. Pecjak, Factorization and momentum-space resummation in deep-inelastic scattering, JHEP 01 (2007) 076 [hep-ph/0607228] [INSPIRE].

[35] T. Becher, M. Neubert and G. Xu, Dynamical threshold enhancement and resummation in Drell-Yan production, JHEP 07 (2008) 030 [arXiv:0710.0680] [INSPIRE].

[36] S. AbdusSalam et al., Benchmark models, planes, lines and points for future SUSY searches at the LHC, Eur. Phys. J. C 71 (2011) 1835 [arXiv:1109.3859] [InSPIRE].

[37] G.L. Kane and J. Leveille, Experimental constraints on gluino masses and supersymmetric theories, Phys. Lett. B 112 (1982) 227 [INSPIRE].

[38] P. Harrison and C. Llewellyn Smith, Hadroproduction of supersymmetric particles, Nucl. Phys. B 213 (1983) 223 [Erratum ibid. B 223 (1983) 542] [INSPIRE].

[39] S. Dawson, E. Eichten and C. Quigg, Search for supersymmetric particles in hadron-hadron collisions, Phys. Rev. D 31 (1985) 1581 [INSPIRE]. 
[40] A. Martin, W. Stirling, R. Thorne and G. Watt, Parton distributions for the LHC, Eur. Phys. J. C 63 (2009) 189 [arXiv:0901.0002] [inSPIRE].

[41] M. Beneke, M. Czakon, P. Falgari, A. Mitov and C. Schwinn, Threshold expansion of the $g g(q \bar{q}) \rightarrow Q \bar{Q}+X$ cross section at $O\left(\alpha_{s}^{4}\right)$, Phys. Lett. B $690(2010) 483$ [arXiv:0911.5166] [INSPIRE].

[42] Y. Kats and M.D. Schwartz, Annihilation decays of bound states at the LHC, JHEP 04 (2010) 016 [arXiv:0912.0526] [INSPIRE].

[43] V.S. Fadin and V.A. Khoze, Threshold behavior of heavy top production in $e^{+} e^{-}$collisions, JETP Lett. 46 (1987) 525 [INSPIRE].

[44] A. Hoang et al., Top-anti-top pair production close to threshold: synopsis of recent NNLO results, Eur. Phys. J. direct C 2 (2000) 1 [hep-ph/0001286] [InSPIRE].

[45] M. Beneke, A. Signer and V.A. Smirnov, Top quark production near threshold and the top quark mass, Phys. Lett. B 454 (1999) 137 [hep-ph/9903260] [INSPIRE].

[46] I.I. Bigi, V.S. Fadin and V.A. Khoze, Stop near threshold, Nucl. Phys. B 377 (1992) 461 [INSPIRE].

[47] M.R. Kauth, J.H. Kuhn, P. Marquard and M. Steinhauser, Gluinonia: energy levels, production and decay, Nucl. Phys. B 831 (2010) 285 [arXiv:0910.2612] [INSPIRE].

[48] J.E. Younkin and S.P. Martin, QCD corrections to stoponium production at hadron colliders, Phys. Rev. D 81 (2010) 055006 [arXiv:0912.4813] [INSPIRE].

[49] T. Becher and M. Neubert, Infrared singularities of QCD amplitudes with massive partons, Phys. Rev. D 79 (2009) 125004 [Erratum ibid. D 80 (2009) 109901] [arXiv:0904.1021] [INSPIRE].

[50] G. Bozzi, B. Fuks and M. Klasen, Non-diagonal and mixed squark production at hadron colliders, Phys. Rev. D 72 (2005) 035016 [hep-ph/0507073] [INSPIRE].

[51] S. Cassel, Sommerfeld factor for arbitrary partial wave processes, J. Phys. G 37 (2010) 105009 [arXiv: 0903.5307] [inSPIRE].

[52] H. Baer, V. Barger, A. Lessa and X. Tata, LHC discovery potential for supersymmetry with $\sqrt{s}=7 \mathrm{TeV}$ and 5-30 fb ${ }^{-1}$, Phys. Rev. D 85 (2012) 051701 [arXiv:1112.3044] [INSPIRE].

[53] NLL-fast, available at http://web.physik.rwth-aachen.de/service/wiki/bin/view/Kraemer/SquarksandGluinos.

[54] A. Djouadi, M. Muhlleitner and M. Spira, Decays of supersymmetric particles: the program SUSY-HIT (SUspect-SdecaY-HDECAY-InTerface), Acta Phys. Polon. B 38 (2007) 635 [hep-ph/0609292] [INSPIRE].

[55] A. Djouadi, J.-L. Kneur and G. Moultaka, SuSpect: a Fortran code for the supersymmetric and Higgs particle spectrum in the MSSM, Comput. Phys. Commun. 176 (2007) 426 [hep-ph/0211331] [INSPIRE].

[56] M. Bonvini, S. Forte, M. Ghezzi and G. Ridolfi, Threshold resummation in SCET vs. perturbative QCD: an analytic comparison, Nucl. Phys. B 861 (2012) 337 [arXiv: 1201.6364] [INSPIRE].

[57] C.W. Bauer, S. Fleming, D. Pirjol and I.W. Stewart, An effective field theory for collinear and soft gluons: heavy to light decays, Phys. Rev. D 63 (2001) 114020 [hep-ph/0011336] [INSPIRE]. 
[58] C.W. Bauer, D. Pirjol and I.W. Stewart, Soft collinear factorization in effective field theory, Phys. Rev. D 65 (2002) 054022 [hep-ph/0109045] [INSPIRE].

[59] M. Beneke, A. Chapovsky, M. Diehl and T. Feldmann, Soft collinear effective theory and heavy to light currents beyond leading power, Nucl. Phys. B 643 (2002) 431 [hep-ph/0206152] [inSPIRE].

[60] M. Beneke and T. Feldmann, Multipole expanded soft collinear effective theory with non-abelian gauge symmetry, Phys. Lett. B 553 (2003) 267 [hep-ph/0211358] [INSPIRE].

[61] A. Pineda and J. Soto, Effective field theory for ultrasoft momenta in NRQCD and NRQED, Nucl. Phys. Proc. Suppl. 64 (1998) 428 [hep-ph/9707481] [INSPIRE].

[62] M. Beneke, New results on heavy quarks near threshold, hep-ph/9806429 [INSPIRE].

[63] N. Brambilla, A. Pineda, J. Soto and A. Vairo, Potential NRQCD: an effective theory for heavy quarkonium, Nucl. Phys. B 566 (2000) 275 [hep-ph/9907240] [INSPIRE].

[64] M. Beneke, Perturbative heavy quark-anti-quark systems, in the proceedings of the $8^{\text {th }}$ International Symposium on Heavy Flavor Physics (Heavy Flavors 8), July 25-29, Southampton, U.K. (1999), hep-ph/9911490 [INSPIRE].

[65] G.T. Bodwin, E. Braaten and G.P. Lepage, Rigorous QCD predictions for decays of P wave quarkonia, Phys. Rev. D 46 (1992) 1914 [hep-lat/9205006] [INSPIRE].

[66] C.W. Bauer, S. Fleming, D. Pirjol, I.Z. Rothstein and I.W. Stewart, Hard scattering factorization from effective field theory, Phys. Rev. D 66 (2002) 014017 [hep-ph/0202088] [INSPIRE]. 